\title{
Neutrino pair annihilation near accreting, stellar-mass black holes
}

\author{
R. Birkl ${ }^{1}$, M. A. Aloy ${ }^{1,2}$, H.-Th. Janka ${ }^{1}$, and E. Müller ${ }^{1}$ \\ 1 Max-Planck-Institut für Astrophysik, Postfach 1317, 85741 Garching, Germany \\ e-mail: rbirkl@mpa-garching.mpg.de \\ 2 Departamento de Astronomía y Astrofísica, Universidad de Valencia, 46100 Burjassot, Spain
}

Received 23 August 2006 / Accepted 14 November 2006

\begin{abstract}
Context. We investigate the deposition of energy and momentum due to the annihilation of neutrinos $(v)$ and antineutrinos $(\bar{v})$ in the vicinity of steady, axisymmetric accretion tori around stellar-mass black holes (BHs). This process is widely considered as an energy source for driving ultrarelativistic outflows with the potential to produce gamma-ray bursts.

Aims. We analyze the influence of general relativistic (GR) effects in combination with different neutrinosphere properties on the $v \bar{v}$-annihilation efficiency and spatial distribution of the energy deposition rate.

Methods. Assuming axial symmetry, we numerically compute the annihilation rate 4-vector. For this purpose, we construct the local neutrino distribution by ray-tracing neutrino trajectories in a Kerr space-time using null geodesics. We vary the value of the dimensionless specific angular momentum $a$ of the central BH, which provides the gravitational field in our models. We also study different shapes of the neutrinospheres, spheres, thin disks, and thick accretion tori, whose structure ranges from idealized tori to equilibrium non-selfgravitating matter distributions. Furthermore, we compute Newtonian models where the influence of the gravitational field on the annihilation process is neglected.

Results. Compared to Newtonian calculations, GR effects increase the total annihilation rate measured by an observer at infinity by a factor of two when the neutrinosphere is a thin disk, but the increase is only $\approx 25 \%$ for toroidal and spherical neutrinospheres. Comparing cases with similar luminosities, thin disk models yield the highest energy deposition rates by $v \bar{v}$-annihilation, and spherical neutrinospheres the lowest ones, independently of whether GR effects are included. Increasing $a$ from 0 to 1 enhances the energy deposition rate measured by an observer at infinity by roughly a factor of 2 due to the change of the inner radius of the neutrinosphere. General relativity and rotation cause important differences in the spatial distribution of the energy deposition rate by $v \bar{v}$-annihilation.
\end{abstract}

Key words. gamma rays: bursts - neutrinos - accretion, accretion disks - relativity - black hole physics - stars: neutron

\section{Introduction}

It is widely believed that systems powering gamma-ray bursts (GRB) could be newborn, stellar-mass black holes (BHs) accreting matter at hyper-critical rates (up to several solar masses per second) from a surrounding accretion disk with a mass of some hundredth of a solar mass up to possibly a solar mass (see, e.g., Piran 2005). These central engines may form in a "collapsar" event where the core of a massive, rotating Wolf-Rayet star collapses to a $\mathrm{BH}$ and the accretion of the stellar envelope may eventually lead to a GRB-supernova event with relativistic mass ejection along the rotation axis (Woosley 1993; MacFadyen \& Woosley 1999; Aloy et al. 2000). Accreting BHs may also be the remnants of mergers of two compact objects in close binaries (Eichler et al. 1989; Mochkovitch et al. 1993). In the first scenario the system is embedded in the envelope of the progenitor star, and the accretion disk is fed by stellar matter yielding very long ( 10-1000 s) accretion time scales comparable to the collapse time scale of the progenitor star. In the second scenario viscous transport in the accretion torus sets the secular time scale of the system $(\sim 0.01-1 \mathrm{~s})$ formed during the merger.

The conditions in the vicinity of steady-state, hyperaccreting BHs have been analytically studied by Jaroszynski (1993, 1996); Popham et al. (1999); Narayan et al. (2001); Di Matteo et al. (2002); Kohri \& Mineshige (2002); Chen \& Beloborodov (2006), who determined the efficiency of energy loss by neutrino emission and the efficiency of energy conversion by neutrino-antineutrino $(v \bar{v})$ pair annihilation into electrons and positrons. Three-dimensional hydrodynamic simulations have explored the time-dependent accretion in $\mathrm{BH}$-torus systems, which are the remnants of neutron star-neutron star (NS+NS) and NS+BH mergers (Ruffert \& Janka 1999; Setiawan et al. 2004; Lee et al. 2004, 2005a,b). These investigations considered a rather compact torus (typical size: $\sim 10-20$ Schwarzschild radii) containing between a few hundredth of a solar mass and some $0.1 M_{\odot}$. Such torus masses result from NS+NS and NS+BH merger simulations (Janka et al. 1999; Ruffert \& Janka 1999; Janka \& Ruffert 2002; Rosswog et al. 2003; Shibata et al. 2003, 2005; Oechslin \& Janka 2006). The torus is partly opaque to neutrinos because of its high density. Typically, neutrino luminosities in excess of $10^{53} \mathrm{erg} \mathrm{s}^{-1}$ are produced. Under these conditions, the reactions $v+\bar{v} \rightarrow \mathrm{e}^{+}+\mathrm{e}^{-} \rightarrow \gamma+\gamma$ give rise to energy deposition in the close vicinity of the $\mathrm{BH}$ at rates ranging from several $10^{49} \mathrm{erg} \mathrm{s}^{-1}$ up to more than $10^{51} \mathrm{erg} \mathrm{s}^{-1}$ (Ruffert \& Janka 1999; Janka et al. 1999; Setiawan et al. 2004, 2006). The resulting $\mathrm{e}^{+} \mathrm{e}^{-}$-pair plasma-photon fireball may power an ultrarelativistic outflow of baryons with typical Lorentz factors of $10^{2}-10^{3}$, provided the baryon loading (i.e., the baryon rest mass compared to the internal energy in the outflow) remains sufficiently low (see, e.g., Aloy et al. 2005, and references therein).

Several studies involving different levels of sophistication have been performed, in order to determine the amount of 
energy which can be released by $v \bar{v}$-annihilation near a (rotating) stellar-mass $\mathrm{BH}$.

Jaroszynski (1993, 1996) investigated stationary configurations consisting of massive, dense and non-selfgravitating tori with different angular momentum distributions and different specific entropies, orbiting stellar-mass Kerr BHs. In particular, he considered the neutrino emission of isentropic tori in the external field of Kerr BHs as an approximation of what results from merger events or failed supernovae. Using the neutrino opacities of Burrows \& Lattimer (1986) and assuming that the neutrinos have an equilibrium (Fermi-Dirac) distribution given by the temperature and chemical potential at the neutrinosphere, Jaroszynski (1996) determined the neutrino radiation field at a given point by following backwards null geodesics in Kerr spacetime until a point is reached in the torus where the neutrino optical depth is $\tau_{v} \approx 1$. He found that the energy deposition rate due to $v \bar{v}$-annihilation increases with increasing entropy and with increasing spin of the Kerr $\mathrm{BH}$. He also claimed that $\mathrm{BH}$-torus configurations resulting from $\mathrm{NS}+\mathrm{NS}$ merger events do not provide sufficient energy to likely be sources of GRBs.

Extending earlier Newtonian calculations of Cooperstein et al. (1986) and Goodman et al. (1987), Salmonson \& Wilson (1999) analytically determined the proper energy deposition rate per unit proper time by $v \bar{v}$-annihilation near the surface of a spherical NS (the neutrinosphere was assumed to coincide with the NS surface) including general relativistic (GR) effects. They concluded that the inclusion of the GR effects of ray bending and redshift (neutrino trajectories were computed in the Schwarzschild metric) enhances the proper deposition rate per unit of proper time compared to the Newtonian values by up to a factor of 4 for a neutrinosphere located at 2.5 Schwarzschild radii relevant for a proto-NS. An enhancement factor of 30 is possible, if the radius of the neutrinosphere shrinks to 1.5 Schwarzschild radii, which happens during the collapse of a NS to a $\mathrm{BH}$.

Asano \& Fukuyama (2000) studied the influence of GR effects on the $v \bar{v}$-annihilation rate assuming two different geometries of the $v \bar{v}$-emitting regions: a spherically symmetric emission region, and a thin disk emitter surrounding a Schwarzschild BH. The spherical geometry was already studied by Salmonson \& Wilson (1999), but Asano \& Fukuyama (2000) improved on this work by approximately taking into account that some fraction of the deposited energy might not escape from the gravitational potential well, and thus cannot contribute to power GRBs. For disk-shaped neutrinospheres they determined the energy deposition rate per unit world time for an observer located at infinity by computing the annihilation rate near the symmetry axis. The latter restriction allows for a semianalytic treatment by making use of the axial symmetry of the neutrino source. Contrary to Salmonson \& Wilson (1999), they found that GR effects do not substantially change the energy deposition rate, neither for the spherically symmetric case nor for the disk case. This discrepancy arises, because the energy deposition rates were calculated with different constraints in both investigations. Salmonson \& Wilson (1999) considered the proper energy deposition rate per unit proper time, which is enhanced by the GR effects of gravitational blueshift and bending of trajectories, for a fixed neutrino luminosity at infinity, while Asano \& Fukuyama (2000) computed the (local) energy deposition rate per unit world time by assuming a given value of the effective temperature of the neutrinosphere and thus of the neutrinosphere luminosity. The inverse redshift factor relating the local luminosity to the luminosity at infinity explains the enhancement of the rate in case of Salmonson \& Wilson (1999). However, as argued by Asano \& Fukuyama (2000), the neutrinosphere luminosity, which is restricted or provided by models of the GRB engine, is the appropriate quantity to use when determining the influence of GR effects on the $v \bar{v}$-annihilation process.

Improving on their earlier work, Asano \& Fukuyama (2001) considered a non-selfgravitating, geometrically infinitely thin accretion disk surrounding a Kerr BH. The disk was either assumed to be isothermal or to have a prescribed temperature gradient. They found that if the energy deposition (along the rotation axis) is mainly due to neutrinos from the central part of the disk near the horizon, redshift effects are dominant, and the energy deposition rate is consequently reduced compared to the case, in which GR effects were neglected during the computation. Instead, if neutrinos that are emitted at larger radii dominate the energy deposition rate, GR bending effects become important. This causes a GR enhancement of the energy deposition rate by a factor of about 2, irrespective of the spin of the Kerr BH.

Miller et al. (2003) considered the full three-dimensional problem of numerically computing the $v \bar{v}$-annihilation around a Kerr $\mathrm{BH} /$ thin accretion disk system using the full geodesic equations. They determined the energy-momentum deposition rate 4-vector per unit 4-volume in a given local observer's orthonormal frame. From this covariant quantity they derived the (coordinate-dependent, i.e. nonconvariant) energy-momentum deposition rate per proper time in Boyer-Lindquist coordinates for their observer, which is an adequate approximation to the 4-momentum per proper time at the observer's location as long as the observer is not too close to the horizon of the BH. Miller et al. (2003) imaged the accretion disk for a specified, but arbitrary off-axis observer. They did this by integrating geodesics along the Boyer-Lindquist energy-momentum vectors until they either hit the $\mathrm{BH}$, the disk, or reach $r=50 \mathrm{M}$. They confirmed the findings of Asano \& Fukuyama (2001) as to an only moderate GR enhancement of the energy deposition rate near the rotation axis, but they also showed that the dominant contribution to the energy deposition rate comes from near the surface of the disk, where the rate is a factor 10-20 times larger than on the rotation axis. This enhancement of the rate is independent of the spin of the BH. Miller et al. (2003) performed their analysis for five Kerr BHs with different angular momenta, and for each case they binned the Boyer-Lindquist time component of the energy-momentum deposition rate 4-vector reaching $r=50 \mathrm{M}$ into 20 polar angle bins. The resulting energy-momentum deposition rate per unit solid angle (as a function of polar angle) peaks along the surface of a cone centered on the rotation axis. The cone has a half-opening angle of $\pi / 4$, because the spatial components of the energy-momentum deposition rate 4-vector are tilted by about 45 degrees towards the rotation axis near the surface of the disk. The total (integrated over polar angle) energy-momentum deposition rate approximately varies linearly with the spin of the $\mathrm{BH}$, the energy deposition rate of a maximally rotating Kerr $\mathrm{BH}$ being about twice that of a non-rotating Schwarzschild $\mathrm{BH}$. This is in rough agreement with the results of Jaroszynski (1993).

The previous studies discussed above were concerned with a restricted set of specific, idealized cases: spherical neutrinospheres (Salmonson \& Wilson 1999; Asano \& Fukuyama 2000), geometrically infinitely thin disks and hence neutrinospheres (Asano \& Fukuyama 2000, 2001; Miller et al. 2003), and specifically designed sequences of torus models without the possibility to identify the separate influence of different components and properties of the neutrino emitting system (Jaroszynski 1993, 1996). In the following we present a more comprehensive and systematic study of energy-momentum deposition by 
$v \bar{v}$-annihilation. We analyze how the neutrino distribution and the resulting $v \bar{v}$-annihilation are influenced by GR effects, the geometry and the properties of the neutrinosphere, and the mass and spin of the central BH. To this end, we examine the most likely configurations occurring in compact astrophysical systems: spherical neutrinospheres present in NSs, and thin disks and thick tori surrounding stellar-mass BHs. We explicitly remark that the absolute values of the neutrino luminosity and of the energy-momentum deposition rate by $v \bar{v}$-annihilation do not play an important role for the discussion in this paper. Due to the simplicity of the neutrino source models considered here, our numbers for these quantities should not be interpreted quantitatively. They sensitively depend on the location and temperature of the neutrinospheres, which must be expected to be different in detailed hydrodynamic simulations that include some treatment of the neutrino transport. However, for the purpose of comparing the different effects, on which this paper focuses, only the relative changes of the neutrino luminosity and $v \bar{v}$-annihilation matter.

The paper is organized as follows: in Sect. 2 we discuss the theoretical fundamentals for calculating the $v \bar{v}$-annihilation rate in a given Kerr spacetime, and how we construct equilibrium accretion tori surrounding Kerr BHs. In Sect. 3 we give a description of the numerical implementation of the ray-tracing algorithm used to calculate the annihilation rate. The results of our parameter study are discussed in Sect. 4, and the conclusions of our work are presented in Sect. 5. Finally, technical details concerning the calculation of the annihilation rate are given in Appendix A, and convergence tests performed on our ray-tracing algorithm are presented in Appendix B.

\section{Theoretical fundamentals}

Unless stated otherwise, we use geometrized units throughout this paper, so that $c=G=1$, where $c$ is the speed of light in vacuum and $G$ is Newton's gravitational constant. Greek and Roman indices denote spacetime components (0-3) and spatial components (1-3) of 4-vectors, respectively. The signature of the metric is chosen to be $(+,-,-,-)$, and 3 -vectors are denoted by a bar above the respective symbol, e.g. $\bar{A}$.

\subsection{Calculation of the annihilation rate}

In order to compute the deposition of 4-momentum $Q_{i}^{\alpha}(t, \bar{x})$ by the annihilation of neutrinos and antineutrinos of flavor $i$ with $i \in\{\mathrm{e}, \mu, \tau\}$ into $\mathrm{e}^{+} \mathrm{e}^{-}$-pairs at a spatial point $\bar{x}$ per unit of time and unit of volume, we follow a formalism very close to that of Miller et al. (2003).

In flat spacetime the local annihilation rate is computed from the Lorentz invariant neutrino and antineutrino phase space distribution functions $f_{v_{i}}=f_{v_{i}}(t, \bar{x}, \bar{p})$ and $f_{\bar{v}_{i}}=f_{\bar{v}_{i}}\left(t, \bar{x}, \bar{p}^{\prime}\right)$ (for their exact definition see Appendix A) by the following integral

$Q_{i}^{\alpha}=\int \mathrm{d}^{3} p \mathrm{~d}^{3} p^{\prime} A_{i}^{\alpha}\left(\bar{p}, \bar{p}^{\prime}\right) f_{v_{i}} f_{\bar{v}_{i}}$,

where the $A_{i}^{\alpha}$ are functions defined in Appendix A. They are based on a covariant generalization of the expressions given in Ruffert et al. (1997). Note that although we are concerned only with time-independent models here, we include the argument $t$ in the distribution functions $f_{v_{i}}$ and $f_{\bar{v}_{i}}$ for the sake of generality. In the following we omit the indices of $f$.

Except for minor redefinitions Eq. (1) can also be used in curved spacetimes (Miller et al. 2003), because the annihilation process, which is a microphysical phenomenon, happens so

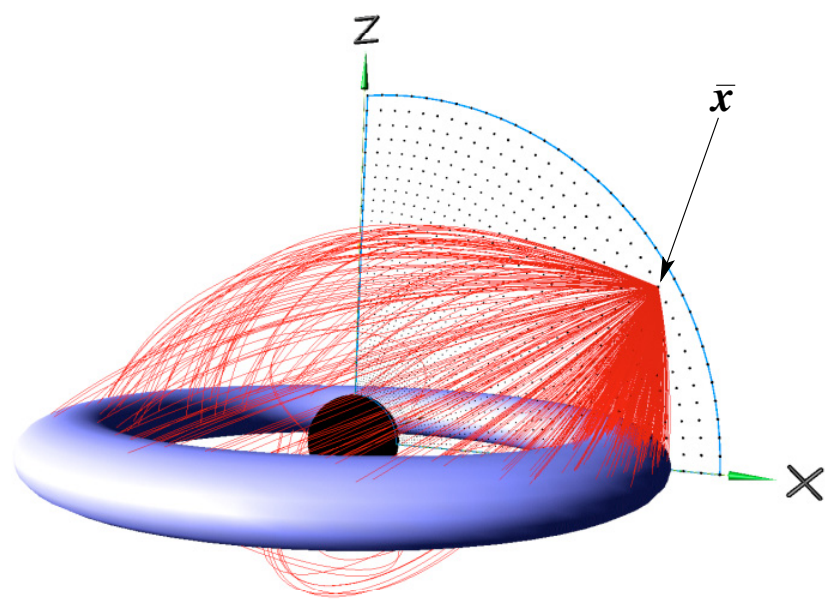

Fig. 1. Ray-tracing of neutrinos in a Kerr BH spacetime. At every point $\bar{x}$ where the annihilation rate is to be calculated, geodesics (red curves) arriving from random directions are traced back until they hit the neutrinosphere (blue torus). The computational grid is marked by the small black dots and its boundary by the blue circle. See the electronic edition for a color version of the figure.

rapidly and on such small length scales that the effects of stellar gravitational fields can be safely neglected. Gravity affects only the propagation of the (anti)neutrinos between their emission (or emergence from the neutrinosphere) and their annihilation.

In our models the gravitational field, which leads to ray bending and redshift, is provided by the central Kerr BH of mass $M$ and (dimensionless) angular momentum parameter $a \equiv$ $J / M^{2}$ (where $J$ is the angular momentum of the $\mathrm{BH}$, and $0 \leq$ $a \leq 1)$, whose metric is given in Boyer-Lindquist coordinates $(t, r, \theta, \phi)$ by

$\mathrm{d} s^{2}=g_{t t} \mathrm{~d} t^{2}+g_{\phi \phi} \mathrm{d} \phi^{2}+2 g_{t \phi} \mathrm{d} t \mathrm{~d} \phi+g_{r r} \mathrm{~d} r^{2}+g_{\theta \theta} \mathrm{d} \theta^{2}$

with

$g_{t t}=1-\frac{2 M r}{\rho^{2}}$

$g_{\phi \phi}=-\left[r^{2}+M^{2} a^{2}+\frac{2 r M^{3} a^{2} \sin ^{2} \theta}{\rho^{2}}\right] \sin ^{2} \theta$,

$g_{t \phi}=\frac{2 r M^{2} a \sin ^{2} \theta}{\rho^{2}}$,

$g_{r r}=-\frac{\rho^{2}}{\Delta}$

$g_{\theta \theta}=-\rho^{2}$,

where

$\Delta=r^{2}-2 M r+(a M)^{2}$,

$\rho^{2}=r^{2}+(a M)^{2} \cos ^{2} \theta$.

Let us now consider an observer located at the point $\bar{x}$ where the annihilation happens (Fig. 1). We will refer to this observer as the local observer, and the quantities measured in his frame are denoted by the subscript " $L$ ". The local frame is defined by an orthonormal base $\left\{\boldsymbol{e}_{t}, \boldsymbol{e}_{r}, \boldsymbol{e}_{\theta}, \boldsymbol{e}_{\phi}\right\}$ such that a local observer is at rest in the global $(r, \theta, \phi)$ coordinate system, i.e. its 4 -velocity fulfills $u^{a}=0$ (the resulting orthonormal base vectors are given in Appendix A). Hence, one cannot calculate the annihilation rate inside the ergosphere, where no observers can be at rest. This restriction could be lifted by using observers dragged along by the $\mathrm{BH}$, i.e. for observers with $u_{a}=g_{a \beta} u^{\beta}=0$. However, the 
energy released inside the ergosphere will end up in the $\mathrm{BH}$, and thus it is of no relevance for a GRB. We therefore exclude the region inside of the ergosphere from our analysis.

In the immediate surroundings of a point $\bar{x}$ a curved spacetime can be approximated by a tangential flat spacetime. Thus, a local observer can use Eq. (1) to calculate the annihilation rate. Consequently, one has to generalize only the momenta $\bar{p}$ in Eq. (1) to $\bar{p}_{\mathrm{L}}$, in order to compute the annihilation rate $Q_{i}^{\alpha}$ in the frame of a local observer.

For doing so, one also needs to calculate the (anti)neutrino distribution functions in the frame of the local observer $f\left(t, \bar{x}, \bar{p}_{\mathrm{L}}\right)$. We take first the 4-momentum vector components $p_{\mathrm{L}}^{\alpha}=\left(E_{\mathrm{L}}, \bar{p}_{\mathrm{L}}\right)$, with ${ }^{1} E_{\mathrm{L}}=\left|\bar{p}_{\mathrm{L}}\right|$, measured in the local frame and transform them into the components

$p^{\alpha}=p_{\mathrm{L}}^{\beta} \boldsymbol{e}_{\beta}^{\alpha}$

measured in the global Boyer-Lindquist frame. Hence, the spatial components $\bar{p}=p^{a}$ are functions depending on $\bar{p}_{\mathrm{L}}$, such that we get a new distribution function $f(t, \bar{x}, \bar{p})=$ $f\left(t, \bar{x}, \bar{p}_{\mathrm{L}}(\bar{p})\right)$. Assuming now that the (anti)neutrinos do not experience collisions, their propagation is described by the collisionless Boltzmann equation in curved spacetime (see, e.g., Misner et al. 1973)

$\frac{\mathrm{d} f(t(\lambda), \bar{x}(\lambda), \bar{p}(\lambda))}{\mathrm{d} \lambda}=0$,

where $\lambda$ is a parameter along the neutrino path. This equation implies that the distribution function is constant along particle trajectories. Since we consider (anti)neutrinos to be massless, their propagation takes place along null geodesics. The latter are given by

$$
\begin{aligned}
& \frac{\mathrm{d} x^{\alpha}}{\mathrm{d} \lambda}=p^{\alpha}, \\
& \frac{\mathrm{d} p^{\alpha}}{\mathrm{d} \lambda}=-\Gamma_{\beta \gamma}^{\alpha} p^{\alpha} p^{\beta},
\end{aligned}
$$

with $g_{\alpha \beta} p^{\alpha} p^{\beta}=0$ (see, e.g., Riffert et al. 1998). To compute the distribution function $f(t, \bar{x}, \bar{p})$ of either a neutrino or an antineutrino at the spacetime location $(t, \bar{x})$ of momentum $\bar{p}$, we simply use the above equations to trace the path of that (anti)neutrino back in time until it hits its neutrinosphere (Fig. 1). In most cases this will never happen, and then $f(t, \bar{x}, \bar{p})=0$. In the other cases, we get an emission event at $\left(t_{\mathrm{E}}, \bar{x}_{\mathrm{E}}\right)$ with a neutrino 4-momentum $p_{\mathrm{E}}^{\alpha}=\left(E_{\mathrm{E}}, \bar{p}_{\mathrm{E}}\right)$. Hence, using Eq. (3) this implies

$f(t, \bar{x}, \bar{p})=f\left(t_{\mathrm{E}}, \bar{x}_{\mathrm{E}}, \bar{p}_{\mathrm{E}}\right)$.

Note that $f\left(t_{\mathrm{E}}, \bar{x}_{\mathrm{E}}, \bar{p}_{\mathrm{E}}\right)$ is the distribution function at the emission point in the global frame, and it has to be related to the corresponding function in the frame comoving with the neutrinosphere. This function has the form of a black body for fermions (the chemical potential is neglected, which is justified for the typical conditions met in the systems we are interested in), and therefore it depends only on the comoving frame energy $E_{\mathrm{C}}$. Taking into account that the 4-velocity $u_{\mathrm{C}}\left(t, \bar{x}_{\mathrm{E}}\right)$ of the neutrinosphere is equal to the time-like base vector of the comoving frame, the comoving frame energy $E_{\mathrm{C}}$ is the projection of the 4-momentum $p_{\mathrm{E}}$ onto $u_{\mathrm{C}}\left(t, \bar{x}_{\mathrm{E}}\right)$ :

$E_{\mathrm{C}}=p_{\mathrm{E}} \cdot u_{\mathrm{C}}$

${ }^{1}$ In the local frame $\left|\bar{p}_{L}\right|^{2}=\left(p_{L}^{1}\right)^{2}+\left(p_{L}^{2}\right)^{2}+\left(p_{L}^{3}\right)^{2}$.
Hence, the rhs of Eq. (4) can be expressed in terms of the comoving frame distribution function as

$f\left(t_{\mathrm{E}}, \bar{x}_{\mathrm{E}}, \bar{p}_{\mathrm{E}}\right)=\frac{1}{1+\exp \left(\frac{E_{\mathrm{C}}}{k_{\mathrm{B}} T_{\mathrm{C}}}\right)}$,

where $k_{\mathrm{B}}=1.381 \times 10^{-16} \mathrm{erg} \mathrm{\textrm {K } ^ { - 1 }}$ is the Boltzmann constant, and $T_{\mathrm{C}}$ the temperature of the neutrinosphere.

\subsection{Neutrinospheres}

The conditions at the neutrinosphere are of importance when calculating the neutrino and antineutrino luminosities and $v \bar{v}$-annihilation, because the neutrino emission properties sensitively depend on the neutrinosphere temperature $T_{\mathrm{C}}$, and on the radiating area. We therefore investigate various neutrinosphere geometries, constructed in two different ways. In the first approach, we prescribe the location and the shape of the neutrinosphere for several idealized $\mathrm{BH}$-accretion disk systems and study their influence on the annihilation rate. In the second, more elaborate approach we proceed similar to Jaroszynski (1993, 1996), and model the accretion disk as a non-selfgravitating, stationary, geometrically thick equilibrium torus rotating around a Kerr BH with mass $M$ and rotation parameter $a$.

The distribution of the specific enthalpy of the equilibrium tori is computed according to the method of Abramowicz et al. (1978) for disks with uniform specific angular momentum $(l)$. Our equilibrium torus models are constructed by fixing their mass $\left(m_{\mathrm{tor}}\right)$ and the value of the inner equatorial radius of the torus (see, e.g., Font \& Daigne 2002), which we choose to be $r_{\text {in }}=4.1 M$ and whose specification replaces the specification of $l$. Once the specific enthalpy distribution is found, we obtain the rest mass density $(\rho)$ distribution using the assumption that the equation of state is barotropic. We further assume that the dimensionless entropy per baryon carried by photons, $s_{\gamma}=4 a_{\gamma}\left(k_{\mathrm{B}} T\right)^{3} /\left(3 \rho / m_{\mathrm{u}}\right)$, is given and uniform, too ${ }^{2}$. This assumption translates into a relation between the density and the temperature $\left(\rho \propto T^{3}\right)$, which allows us to compute the latter. The resulting tori are radiation dominated for values of $s_{\gamma} \gtrsim 1$. In Sect. 4.2 we take $s_{\gamma}=1$ in the tori, which corresponds to total entropies per baryon in the range of 5 to 12 and agrees with the values resulting from detailed hydrodynamic Newtonian simulations of mergers of compact binaries (Ruffert \& Janka 1999; Setiawan et al. 2006). We further assume that matter in the torus consists of free protons, neutrons, $\mathrm{e}^{-}, \mathrm{e}^{+}$, and photons. For the typical conditions in the torus, it is justified to neglect the effects of strong interactions on the equation of state. With the baryon rest-mass density $\rho$, electron fraction $Y_{\mathrm{e}} \equiv\left(n_{\mathrm{e}^{-}}-n_{\mathrm{e}^{+}}\right) / n_{\mathrm{B}}$ (where $n_{\mathrm{B}}$, and $n_{\mathrm{e}^{ \pm}}$are the number densities of baryons, and of electrons and positrons, respectively) and $s_{\gamma}$ given, the thermodynamic state is unambiguously defined. The specification of $Y_{\mathrm{e}}$ is needed to determine the composition of the torus gas such that the neutrino opacities can be calculated, and its value is chosen to be $Y_{\mathrm{e}}=0.1$ in our paper. Therefore, an equilibrium torus is fully specified by the six parameters $M, a, m_{\mathrm{tor}}, s_{\gamma}, r_{\mathrm{in}}$, and $Y_{\mathrm{e}}$.

For the equilibrium torus models the neutrino and antineutrino opacities are computed as in Janka (2001). The neutrino spectrum is assumed to be that of a black body for fermions with zero chemical potential, and the neutrinospheres are determined as surfaces surrounding the $\mathrm{BH}$, at which the optical depth for

\footnotetext{
2 The constants used in the definition of the photon entropy $s_{\gamma}$ are $a_{\gamma}=8 \pi^{5} /\left(15 h^{3} c^{3}\right)=2.082 \times 10^{49} \mathrm{erg}^{-3} \mathrm{~cm}^{-3}, m_{\mathrm{u}}=1.661 \times 10^{-24} \mathrm{~g}$, and $h=6.626 \times 10^{-27} \mathrm{erg} \mathrm{s}$.
} 
equilibration of (anti)neutrinos is $\tau=2 / 3$. The integration to calculate $\tau$ is done along rays parallel to the symmetry axis, assuming neutrinos to have an average energy according to a thermal spectrum with the local temperature. We point out that this approach to estimate the neutrinospheric surfaces differs from that of Jaroszynski (1996), who integrated neutrino trajectories back to the points where $\tau=1$. Our method nevertheless leads to neutrinosphere geometries, which are very similar to those of Jaroszynski (1996).

However, the neutrinosphere concept is only an approximation. Given some matter distribution, e.g. in an accretion torus, the neutrinospheres roughly separate optically thin from optically thick regions. Strictly speaking, there exists no well defined surface where neutrinos decouple instantaneously from the background, because the neutrino opacities depend on the neutrino energy. It is also a crude simplification to assume that there exists a surface, exterior to which neutrinos stream freely, and interior to which they are in equilibrium with matter and diffuse out suffering multiple scattering. Therefore our definition of the neutrinospheres appears sufficiently good to discuss the basic features of $v \bar{v}$-annihilation in the vicinity of accreting tori in rotational equilibrium around BHs, although it is very approximative and does not take into account the local conditions along the curved geodesical paths of neutrinos.

\section{Numerical implementation}

The $v \bar{v}$-annihilation rate is computed on a spherical grid consisting of $N_{\mathrm{r}}$ points in radial and $N_{\theta}$ points in polar $(\theta)$ direction, respectively. Typically, we use $N_{\theta}=100$ uniformly distributed points excluding a small conical region around the rotation axis of the $\mathrm{BH}$ for numerical reasons. The radial grid is non-equidistant, stretching from the $\mathrm{BH}$-horizon to a radius slightly larger than the most distant radial point of the neutrinosphere (Figs. 2, 3, and 6). The radial grid points are distributed according to $r_{i+1}=(1+\Delta \theta) r_{i}$, where $\Delta \theta$ is the angular grid spacing, and $i=1, \ldots, N_{\mathrm{r}}$ (typically $N_{\mathrm{r}}=100$ ). We assume axial and equatorial symmetry.

For each of our models we trace $N_{\text {rays }}=20000$ rays back in time in random directions, starting from every grid point of the spherical grid exterior to the neutrinospheres. The procedure is performed independently for neutrinos and antineutrinos (except when the neutrinospheres are identical). The time integration is performed with a fourth-order adaptive step-size Runge-Kutta method until the neutrino trajectory hits the neutrinosphere. To determine when a neutrinosphere is hit, we have developed the following mesh refinement algorithm. On a coarse grid of $150 \times 150$ zones we label the zones belonging to the optically thick region (interior to the neutrinosphere), and we flag the zones which are crossed by the neutrinosphere (Fig. 1). The latter zones are further refined, such that each of them is covered by $150 \times 150$ fine zones. Again, we label each of the fine zones belonging to the interior of the neutrinosphere. The whole process has to be performed only once (before calculating the annihilation rate on the spherical grid). This algorithm allows one at each step of the ray-tracing to very rapidly check whether the optically thick region has been reached. In that case the last twenty steps of the ray-tracing are redone with improved accuracy. The described method can deal with arbitrary (axisymmetric) neutrinosphere geometries, but it is very inefficient when applied to cases involving infinitely thin disks. For these cases our algorithm branches to a different "hit-detection" procedure, and checks whether the ( $x, y)$-plane (i.e., the equatorial plane), containing the neutrinosphere and the thin disk, is intersected between the inner and outer radial edge of the disk. Most raytraced neutrinos never hit the neutrinosphere, because either they are trapped by the $\mathrm{BH}$ or because they escape to infinity.

\section{Simulation results}

Although we included all three (anti)neutrino flavors in the previous theoretical discussion, we consider the annihilation of only $v_{\mathrm{e}}$ and $\bar{v}_{\mathrm{e}}$ in our simulations, since the energy deposition is dominated by the latter process. The reasons for that are the reduced production and emission of heavy-lepton neutrinos by GRB accretion tori (see, e.g., Ruffert \& Janka 1999) and the fact that the weak coupling constants for $v \bar{v}$-annihilation of muon and tau neutrinos are roughly a factor of five lower than those of electron-type neutrinos. Thus, in the following we use the shorthand notation $Q^{\alpha}$ for the annihilation rate $Q_{\mathrm{e}}^{\alpha}$ measured in the frame of a local observer.

In order to quantify the total amount of energy released by $v \bar{v}$-annihilation per unit of time, we consider four energy deposition rates, which are all based on volume integrals of the energy component $Q^{t}$ of the annihilation rate 4 -vector. The first two rates are integrals of the rates measured in the local frames:

$\dot{E}_{\nu \bar{v}}^{\text {tot }}=\int_{\mathrm{V}_{\text {tot }}} \mathrm{d} r \mathrm{~d} \theta \mathrm{d} \phi \sqrt{-\operatorname{det}\left(g_{a b}\right)} Q^{t}$,
$\dot{E}_{\nu \bar{v}}^{\text {up }}=\int_{\mathrm{V}_{\text {up }}} \mathrm{d} r \mathrm{~d} \theta \mathrm{d} \phi \sqrt{-\operatorname{det}\left(g_{a b}\right)} Q^{t}$.

Here $V_{\text {tot }}$ and $V_{\text {up }}$ are the volume of the whole computational grid and the volume of that part of the computational grid where $Q^{r}>0$, respectively. In the "up" region the radial component of the momentum vector of the $\mathrm{e}^{+} \mathrm{e}^{-}$-pair created by $v \bar{v}$-annihilation directs outward. Ignoring hydrodynamic effects of the produced $\mathrm{e}^{+} \mathrm{e}^{-}$-photon plasma (i.e., adopting a "free-particle picture"), the energy deposited in this "up" region is likely to eventually reach a distant observer, i.e. it is the crucial region for our study. In contrast, the energy released between the event horizon and $V_{\text {up }}$ is probably swallowed by the $\mathrm{BH}$.

We further consider the corresponding energy deposition rates measured by an infinitely distant observer (Jaroszynski 1993):

$$
\begin{aligned}
& \dot{E}_{\nu \bar{v}}^{\mathrm{tot}, \infty}=\int_{\mathrm{V}_{\mathrm{tot}}} \mathrm{d} r \mathrm{~d} \theta \mathrm{d} \phi \sqrt{-\operatorname{det}\left(g_{\alpha \beta}\right)} Q^{t}, \\
& \dot{E}_{\nu \bar{v}}^{\mathrm{up}, \infty}=\int_{\mathrm{V}_{\mathrm{up}}} \mathrm{d} r \mathrm{~d} \theta \mathrm{d} \phi \sqrt{-\operatorname{det}\left(g_{\alpha \beta}\right)} Q^{t} .
\end{aligned}
$$

Note that while in Eqs. (5) and (6) the integrands contain the determinant of the 3-metric (Latin indices), Eqs. (7) and (8) involve the determinant of the 4-metric (Greek indices). The latter two energy deposition rates are generally smaller than the corresponding rate computed as the integral of $Q^{t}$ in the local frames, because they include the redshift due to the gravitational field. If GRBs are fueled by the process of $v \bar{v}$-annihilation, $\dot{E}_{v \bar{v}}^{\mathrm{up}, \infty}$ (Eq. (8)) represents a rough estimate for the maximum luminosity of the GRB event.

Two other quantities relevant for analyzing our models are the "local luminosity" radiated from the neutrinosphere,

$L_{v}=\frac{\pi k_{\mathrm{B}}^{4}}{h^{3}} F_{3}(0) \int_{v-\text { sphere }} \mathrm{d} \sigma T_{\mathrm{C}}^{4}$, 

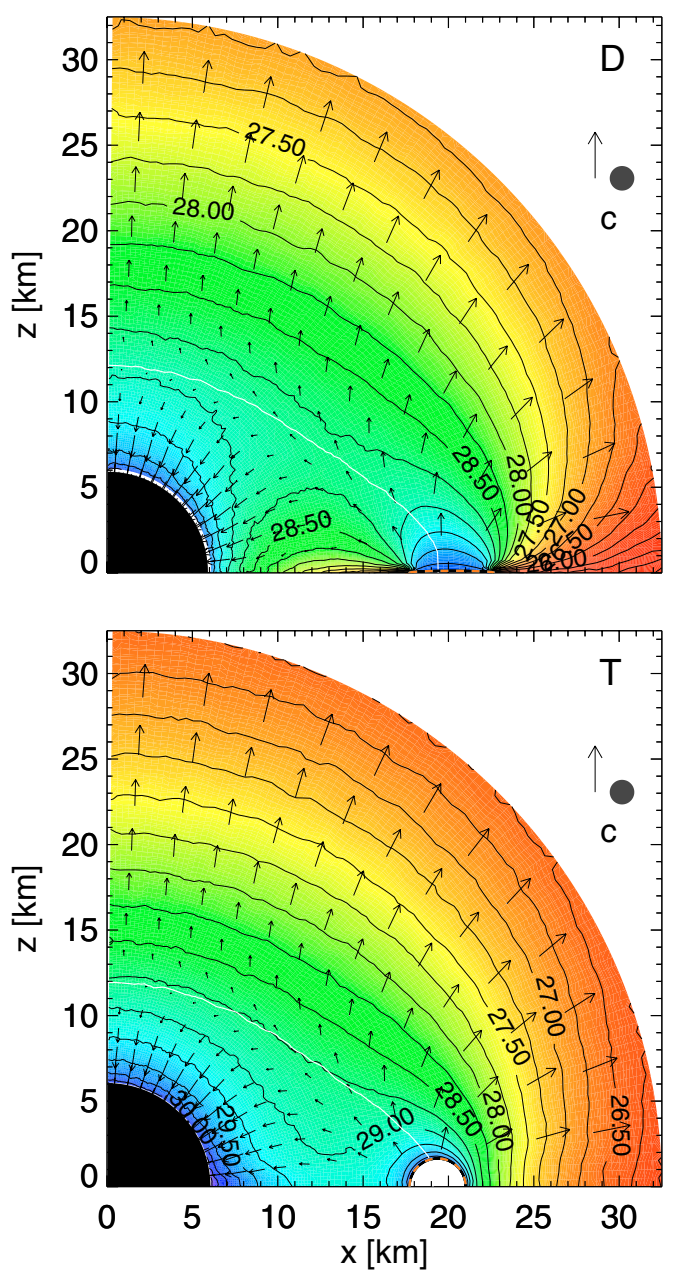
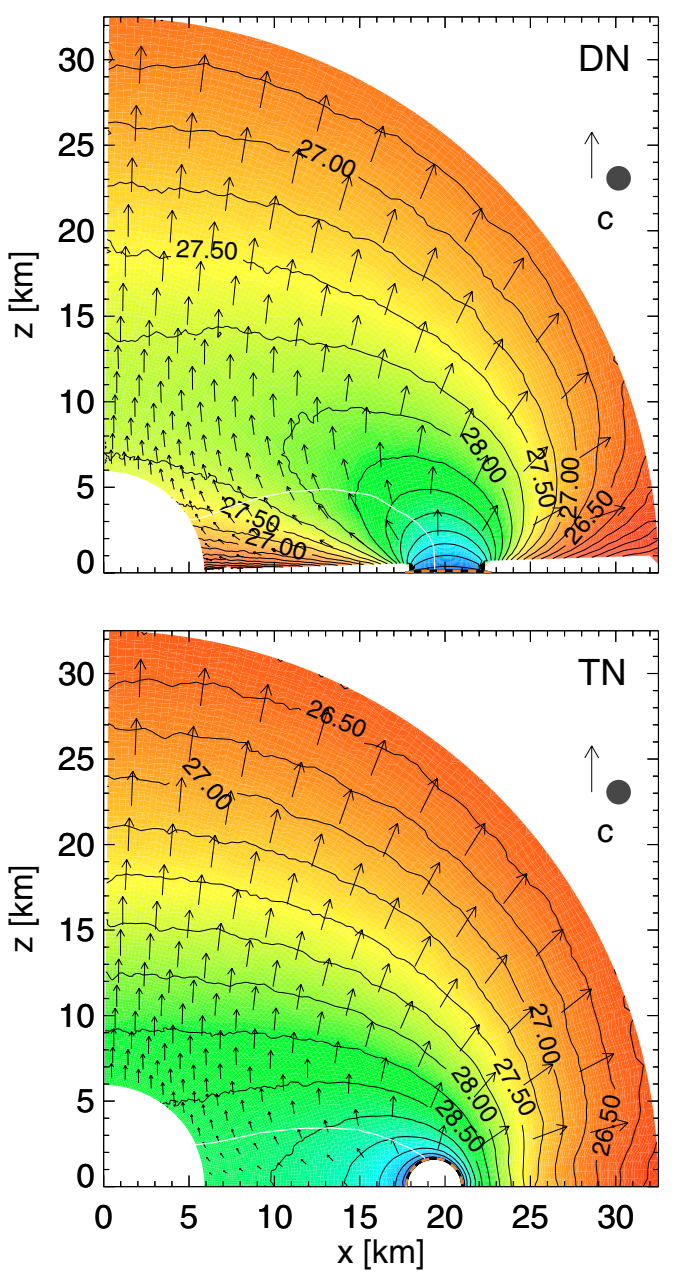

$\lg Q^{t}\left[\operatorname{erg~s}^{-1} \mathrm{~cm}^{-3}\right]$

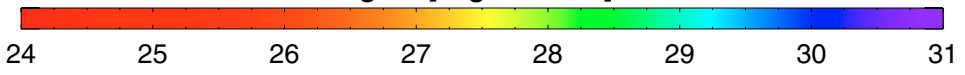

Fig. 2. Annihilation rate 4-vector $Q^{\alpha}$ measured in the frame of a local observer for models with idealized neutrinospheres (see Table 1). The location of the neutrinosphere, which coincides with the antineutrinosphere, is marked by a dashed line. The value of the energy component $Q^{t}$ is color-coded, while the spatial vector $\bar{Q}$ is visualized by showing the spatial velocity vector $\bar{v} \equiv \bar{Q} / Q^{t}$, whose component coplanar (perpendicular) to the displayed $x-z$-plane is given by the black arrows (filled circle). The length (area) of the arrow (filled circle) is a linear measure of the size of the component, the maximum value $(c=1)$ being represented by the arrow (filled circle) in the right upper corner of each panel. Note that for the neutrino evaluation the neutrinosphere is assumed to be non-rotating. Therefore the perpendicular component of $\bar{v}$ is zero here. The big black circular region in the left lower corner of the left two panels represents the $\mathrm{BH}$. In the right two panels the corresponding region is chosen to be white, because these panels show Newtonian models where the GR effects of the BH were disregarded. Finally, in every panel a solid white line separates regions with positive $v^{r}$ from those with negative $v^{r}$. Note that in model DN the white region along the equatorial plane ( $x$-axis) is caused by the saturation of the color scale due to a very low energy deposition rate. See the electronic edition for a color version of the figure.

where we assume blackbody emission from an isotropically radiating surface, and the corresponding "luminosity" for an observer at infinity,

$L_{v}^{\infty}=\frac{\pi k_{\mathrm{B}}^{4}}{h^{3}} F_{3}(0) \int_{v-\text { sphere }} \mathrm{d} \sigma g_{t t} T_{\mathrm{C}}^{4}$.

Here $F_{3}(0)=\int_{0}^{\infty} \mathrm{d} x\left[x^{3} /\left(\mathrm{e}^{x}+1\right)\right]$ and $\mathrm{d} \sigma$ is the appropriate GR surface element of the neutrinosphere. The factor $g_{t t}$ in Eq. (10) accounts for the gravitational redshift. Note that what we call "luminosity" here is actually the total energy emission rate from the neutrinosphere, which is not the quantity directly measured by an observer at a fixed position, because we ignore the direction-dependent variation of the surface area of the neutrinosphere visible to an observer.

The evaluation of the quantities $\dot{E}_{\nu \bar{v}}^{\text {tot, } \infty}$ and $\dot{E}_{\gamma \bar{v}}^{\text {up, } \infty}$ (see Eqs. (7) and (8)) requires two steps. In the first step $Q^{t}$ is calculated at the annihilation point as described in Sect. 2.1, including aberration effects due to the neutrinosphere rotation (for models with $l \neq 0$ ). Using the obtained $Q^{t}$, Eqs. (7) and (8) are evaluated in the second step. Note that these two equations involve an approximation, because the spatial components $Q^{a}$ of the annihilation rate 4 -vector do not enter. This approximation means that the momentum of the $v \bar{v}$-annihilation plasma at the annihilation point is ignored in the transformation of the energy to infinity. In contrast to the evaluation of $Q^{t}$, neutrinosphere aberration effects are neglected in Eq. (10), because this enormously simplifies the 
Table 1. Some properties of the simulated idealized models, whose isothermal neutrinosphere and antineutrinosphere are assumed to coincide and to have the same temperature. The quantities given in the columns of the table from left to right are: the mass $M$ and the (dimensionless) angular momentum parameter $a$ of the $\mathrm{BH}$, the geometry of the (anti)neutrinosphere, the inner and outer radius (disk/torus) or just the radius (sphere) of the neutrinosphere; for toroidal neutrinospheres the ratio of the radial to the vertical diameter of its elliptical meridional cross section is given, too. The other quantities are the Lagrangian angular momentum $l$, measured in terms of the $\mathrm{BH}$ mass $M_{\mathrm{GR}}$ of the GR model, i.e. in case of a GR model we have $M_{\mathrm{GR}}=M \neq 0$, whereas in case of a Newtonian model $(M=0)$ the value of the Lagrangian angular momentum of the corresponding GR model is used (cgs values of $l$ are obtained by applying the conversion factor $\left.\left(M_{\mathrm{GR}} / M_{\odot}\right) \cdot 4.42 \times 10^{15} \mathrm{~cm}^{2} \mathrm{~s}^{-1}\right)$, the comoving frame temperature $T_{\mathrm{C}}$ of the neutrinosphere (in the special model TEMP there is no emission outside of a cylinder around the system axis with radius $8 \mathrm{M}$ ), the total neutrinosphere luminosity $L_{v}^{\infty}=L_{v_{e}}^{\infty}+L_{\bar{v}_{e}}^{\infty}$ for an observer at infinity, the total energy deposition rate, $\dot{E}_{v \bar{\nu}}^{\text {tot }}$, as the integral over the total volume of the local energy deposition rate $Q^{t}$, the total energy deposition rate $\dot{E}_{v \bar{v}}^{\text {up }}$ in the "up" region (defined by $Q^{r}>0$ ), these total energy deposition rates measured by an infinitely distant observer, $\dot{E}_{v \bar{v}}^{\text {tot, } \infty}$ and $\dot{E}_{v \bar{v}}^{\text {up, }, \infty}$, and the efficiencies $q_{v \bar{v}}^{\text {tot, } \infty} \equiv \dot{E}_{v \bar{v}}^{\text {tot, } \infty} / L_{v}^{\infty}$ and $q_{v \bar{v}}^{\text {up, } \infty} \equiv \dot{E}_{v \bar{v}}^{\text {up, } \infty} / L_{v}^{\infty}$, respectively.

\begin{tabular}{|c|c|c|c|c|c|c|c|c|c|c|c|c|c|}
\hline $\begin{array}{c}\text { Model } \\
\text { name }\end{array}$ & $\begin{array}{c}M \\
M_{\odot} \\
\end{array}$ & $a$ & Geometry & $\begin{array}{c}\text { Radii } \\
M \\
\end{array}$ & $\begin{array}{c}l \\
M_{\mathrm{GR}} \\
\end{array}$ & $\begin{array}{c}T_{\mathrm{C}} \\
10^{10} \mathrm{~K} \\
\end{array}$ & $\begin{array}{c}L_{v}^{\infty} \\
10^{52} \mathrm{erg} \mathrm{s}^{-1} \\
\end{array}$ & $\begin{array}{c}\dot{E}_{v \bar{v}}^{\text {tot }} \\
10^{49} \mathrm{erg} \mathrm{s}^{-1} \\
\end{array}$ & $\begin{array}{c}\dot{E}_{\nu \bar{v}}^{\mathrm{up}} \\
10^{49} \mathrm{erg} \mathrm{s}^{-1} \\
\end{array}$ & $\begin{array}{c}\dot{E}_{v \bar{v}}^{\mathrm{tot}, \infty} \\
10^{49} \mathrm{erg} \mathrm{s}^{-1} \\
\end{array}$ & $\begin{array}{c}\dot{E}_{v \bar{v}}^{\mathrm{up}, \infty} \\
10^{49} \mathrm{erg} \mathrm{s}^{-1} \\
\end{array}$ & $\begin{array}{l}q_{\nu \bar{v}, \infty}^{\text {tot }} \\
10^{-3} \\
\end{array}$ & $\begin{array}{l}q_{\nu \bar{v}}^{\text {up }} \\
10^{-3} \\
\end{array}$ \\
\hline $\mathrm{D}$ & 2 & 0 & disk & $6 \leftrightarrow 7.7$ & 0 & 5 & 0.33 & 0.54 & 0.27 & 0.36 & 0.23 & 1.1 & 0.70 \\
\hline $\mathrm{DN}$ & 0 & 0 & disk & $6 \leftrightarrow 7.7$ & 0 & 5 & 0.39 & 0.15 & 0.12 & 0.15 & 0.12 & 0.38 & 0.31 \\
\hline $\mathrm{T}$ & 2 & 0 & torus & $6 \leftrightarrow 7.1 ; 1$ & 0 & 5 & 0.30 & 0.66 & 0.13 & 0.44 & 0.11 & 1.5 & 0.37 \\
\hline TN & 0 & 0 & torus & $6 \leftrightarrow 7.1 ; 1$ & 0 & 5 & 0.39 & 0.16 & 0.095 & 0.16 & 0.095 & 0.41 & 0.24 \\
\hline $\mathrm{S}$ & 2 & 0 & sphere & 3.4 & 0 & 5 & 0.16 & 0.12 & 0.12 & 0.083 & 0.083 & 0.52 & 0.52 \\
\hline SN & 0 & 0 & sphere & 3.4 & 0 & 5 & 0.39 & 0.066 & 0.066 & 0.066 & 0.066 & 0.17 & 0.17 \\
\hline DA1 & 2 & 1 & disk & $6 \leftrightarrow 7.7$ & 0 & 5 & 0.33 & 0.56 & 0.27 & 0.38 & 0.23 & 1.2 & 0.70 \\
\hline DL6.5 & 2 & 0 & disk & $6 \leftrightarrow 7.7$ & 6.5 & 5 & 0.33 & 0.30 & 0.28 & 0.25 & 0.24 & 0.76 & 0.73 \\
\hline DL4 & 2 & 0 & disk & $6 \leftrightarrow 7.7$ & 4 & 5 & 0.33 & 0.38 & 0.24 & 0.28 & 0.21 & 0.85 & 0.62 \\
\hline TL4 & 2 & 0 & torus & $6 \leftrightarrow 7.1 ; 1$ & 4 & 5 & 0.30 & 0.38 & 0.12 & 0.23 & 0.099 & 0.78 & 0.33 \\
\hline DL4N & 0 & 0 & disk & $6 \leftrightarrow 7.7$ & 4 & 5 & 0.39 & 0.13 & 0.11 & 0.13 & 0.11 & 0.32 & 0.29 \\
\hline TL4N & 0 & 0 & torus & $6 \leftrightarrow 7.1 ; 1$ & 4 & 5 & 0.39 & 0.12 & 0.071 & 0.12 & 0.071 & 0.31 & 0.18 \\
\hline REF & 3 & 0 & disk & $6 \leftrightarrow 10$ & 0 & 5 & 2.1 & 8.7 & 5.1 & 6.2 & 4.4 & 2.9 & 2.1 \\
\hline A2B & 3 & 0 & torus & $6.3 \leftrightarrow 9.7 ; 2$ & 0 & 5 & 2.0 & 8.0 & 3.1 & 5.0 & 2.7 & 2.4 & 1.3 \\
\hline $\mathrm{AB}$ & 3 & 0 & torus & $6.7 \leftrightarrow 9.3 ; 1$ & 0 & 5 & 2.0 & 10 & 2.3 & 5.7 & 2.0 & 2.9 & 1.0 \\
\hline A. $5 \mathrm{~B}$ & 3 & 0 & torus & $7.2 \leftrightarrow 8.8 ; 0.5$ & 0 & 5 & 1.9 & 15 & 2.0 & 7.6 & 1.7 & 4.0 & 0.89 \\
\hline SM3 & 3 & 0 & sphere & 5.7 & 0 & 5 & 1.6 & 1.3 & 1.3 & 1.1 & 1.1 & 0.70 & 0.70 \\
\hline RI4.5 & 3 & 0 & disk & $4.5 \leftrightarrow 9.2$ & 0 & 5 & 2.1 & 13 & 6.4 & 8.3 & 5.4 & 4.0 & 2.6 \\
\hline RI3 & 3 & 0 & disk & $3 \leftrightarrow 8.5$ & 0 & 5 & 2.0 & 20 & 6.8 & 11 & 5.5 & 5.6 & 2.8 \\
\hline TEMP & 3 & 0 & torus & $6.7 \leftrightarrow 9.3 ; 1$ & 0 & 6.1 & 2.0 & 53 & 6.9 & 27 & 5.7 & 14 & 2.9 \\
\hline A.5 & 3 & 0.5 & disk & $6 \leftrightarrow 10$ & 0 & 5 & 2.1 & 8.9 & 5.1 & 6.3 & 4.4 & 3.0 & 2.1 \\
\hline A1 & 3 & 1 & disk & $6 \leftrightarrow 10$ & 0 & 5 & 2.1 & 8.9 & 5.1 & 6.5 & 4.4 & 3.1 & 2.1 \\
\hline L2.5 & 3 & 0 & disk & $6 \leftrightarrow 10$ & 2.5 & 5 & 2.1 & 7.7 & 5.0 & 5.7 & 4.2 & 2.7 & 2.0 \\
\hline L4 & 3 & 0 & disk & $6 \leftrightarrow 10$ & 4 & 5 & 2.1 & 6.6 & 4.9 & 5.1 & 4.2 & 2.4 & 2.0 \\
\hline L5 & 3 & 0 & disk & $6 \leftrightarrow 10$ & 5 & 5 & 2.1 & 5.9 & 4.9 & 4.8 & 4.2 & 2.3 & 2.0 \\
\hline
\end{tabular}

evaluation and can be justified by the use of an approximation in Eqs. (7) and (8) as explained above.

\subsection{Idealized models}

In the following we investigate several idealized $\mathrm{BH}$-accretion disk systems. For each of these models the central black hole of mass $M$ and angular momentum $a$ is surrounded by a constructed neutrinosphere (see also Sect. 2.2), which is characterized by the geometry (thin disk, torus, or sphere), the position (radii), the rotation, and the temperature (see Table 1). The rotation of the neutrinosphere is given by the uniform Lagrangian angular momentum $l=-u_{\phi} / u_{t}$ (Abramowicz et al. 1978). This is the GR generalization of the specific angular momentum $l=\bar{x} \times \bar{v}$, where $\bar{x}$ is the position and $\bar{v}$ the velocity. In our models the prescribed geometry and position of the neutrinosphere limit physically reasonable values for the Lagrangian angular momentum $l$ to several times the mass of the $\mathrm{BH}$ (cgs units are obtained by applying the conversion factor $\left(M_{\mathrm{GR}} / M_{\odot}\right) \cdot 4.42 \times$ $10^{15} \mathrm{~cm}^{2} \mathrm{~s}^{-1}$ ), and values of $l \approx 4 M$ approximately describe Keplerian rotation. The neutrinosphere is assumed to be isothermal in the comoving frame, i.e. it has a uniform comoving frame temperature $T_{\mathrm{C}}$. In each idealized model the four parameters of the neutrinosphere presented here are also used for the antineutrinosphere.

The idealized models are constructed for comparison with two reference models, which are the disk models D and REF (see Table 1). Both models have the same temperature $T_{\mathrm{C}}$, and the disk is assumed to be non-rotating $(l=0)$ for computing the $v \bar{v}$-annihilation effects, but the masses $M$ of their non-rotating black holes and their outer disk radii differ. For each reference model there is a series of models (models DN to TL4N for model D and models A2B to L5 for model REF, see Table 1), which are obtained by changing the properties of the corresponding reference model. We explore the influence of the geometry (toroidal models T, A2B, AB, A.5B, and TEMP, and spherical models S, and SM3), of GR effects by studying "Newtonian" models (disk model DN, torus model TN, and sphere model SN), of the inner disk radius (models RI4.5, and RI3), of the black hole angular momentum (models DA1, A.5, and A1), and of the disk rotation (models DL6.5, DL4, TL4, DL4N, TL4N, L2.5, L4, and L5).

For most of the idealized models the effects due to the rotation of the neutrino emitting surface are ignored. Such models correspond to the cases which have $l=0$. Although in astrophysical systems disks and tori always rotate, we nevertheless consider idealized models with no neutrinosphere rotation here, 

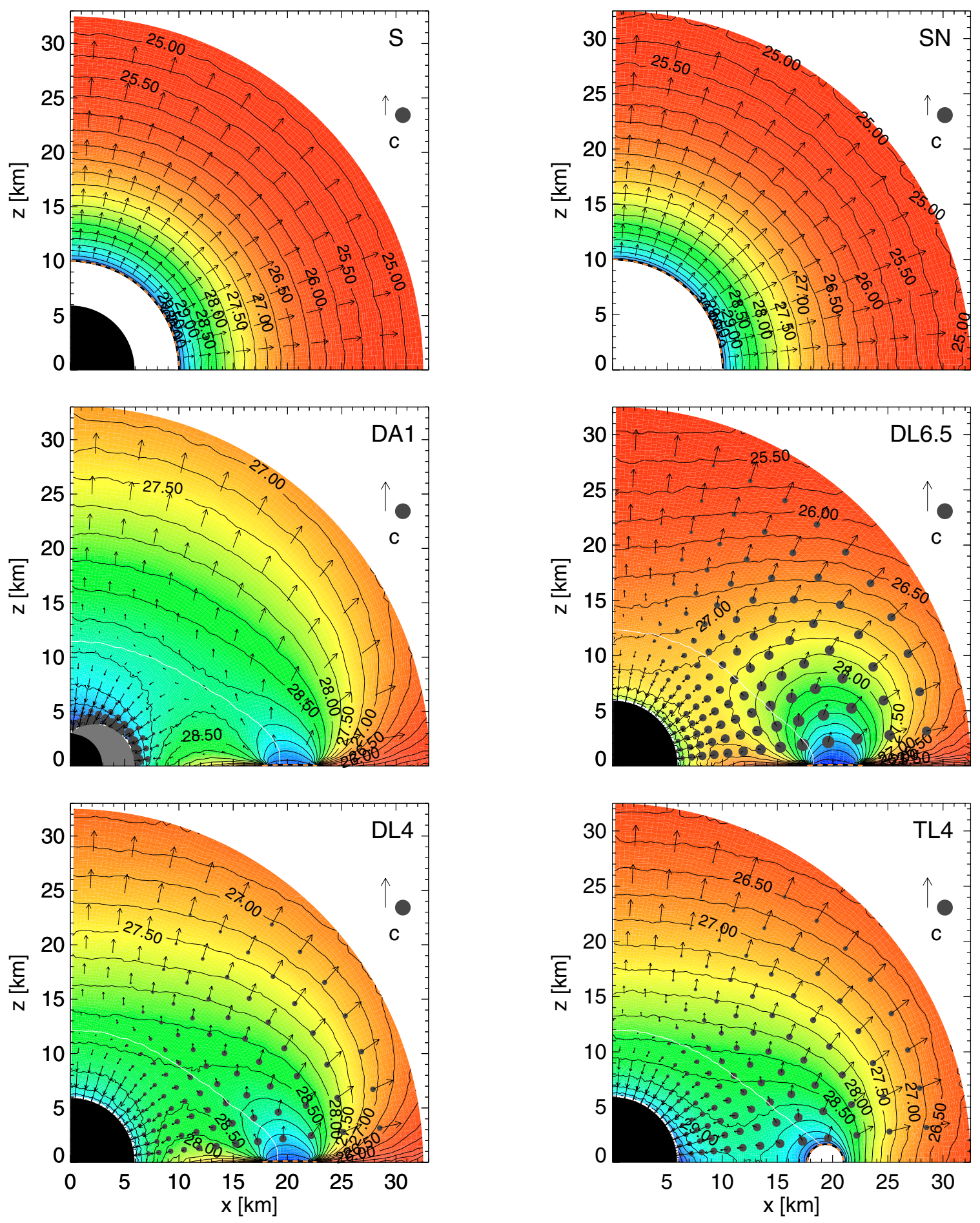

$\lg Q^{t}\left[\operatorname{erg~s}^{-1} \mathrm{~cm}^{-3}\right]$

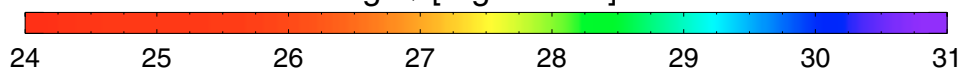

Fig. 3. Same as Fig. 2, but for a different set of models with idealized neutrinosphere geometries (see Table 1). Note that model DA1 contains a central rotating $\mathrm{BH}$ (with $a=1$ ) and therefore possesses an ergosphere outside of the event horizon. This ergosphere is marked by the gray area. See the electronic edition for a color version of the figure.

because we want to selectively study the effects of disk or torus rotation on the neutrino-antineutrino annihilation rate. Later (see Sects. 4.1.3 and 4.1.4), we will demonstrate that the rotation of the neutrino emitting source has a significant effect only on the spatial distribution of the annihilation rate but almost no influence on volume integrated results.

The annihilation rate 4-vector $Q^{\alpha}$ of the series of models D to TL4 is shown in Figs. 2 and 3. Similar plots can also be found 
in Fig. 5 of Miller et al. (2003), and there especially the upper left panel ( $a=0.0$, "MDR") nearly looks like our plot for model DL6.5 (see Fig. 3), where the accretion disk rotates with the super-Keplerian value $l=6.5$. The agreement is even somewhat better in case of model L5 (not visualized in our work), because in contrast to model DL6.5 it has the same inner $(6 M)$ and outer $(10 M)$ disk radii as the mentioned model of Miller et al. (2003). However, we were not able to reproduce the models of Miller et al. (2003) exactly, because of the lack of information about the precise parameters of their models, particularly the rotation law. We also point out that in the pictorial representation of Fig. 5 of Miller et al. (2003), the local value of the azimuthal integral of the "MDR" is provided, while our figures show the local value of $Q^{t}$. If instead of $Q^{t}$ we also compute the azimuthal integral of $Q^{t}$, the contours of, e.g., model DL6.5 become vertical in the vicinity of the rotation axis just like in Miller et al. (2003).

\subsubsection{Influence of general relativity}

In order to study the influence of GR effects, we take the thin disk model $\mathrm{D}$, the torus model $\mathrm{T}$, and the sphere model $\mathrm{S}$, and compare these models with their corresponding Newtonian cases DN, TN, and SN (see Table 1). The Newtonian models have the same neutrinosphere as the corresponding GR models, however, we assume $M=0$, i.e. we neglect the influence of gravity on the neutrino propagation and transformation between observers. The computational grid for a Newtonian model is identical to that of the corresponding GR model, i.e. in particular, the inner radial grid boundary is located at the same radius in both models (just above the horizon of the GR model). All three Newtonian models DN, TN, and SN are assumed to have the same surface area and temperature, and therefore also have the same total (neutrino plus antineutrino) luminosity $L_{v}^{\mathrm{N}}$, i.e. $L_{v, \mathrm{DN}}=L_{v, \mathrm{TN}}=L_{v, \mathrm{SN}}=3.9 \times 10^{51} \mathrm{erg} \mathrm{s}^{-1}($ see Table 1$)$. Although the GR models D, T, and $\mathrm{S}$ have the same metric ( $M=2 M_{\odot}, a=0$ ), a local comoving observer does not measure the same surface areas, because the surfaces of these three models do not coincide such that they are differently affected by the curvature of spacetime. Therefore, despite having the same temperature $\left(T_{\mathrm{C}}=5 \times 10^{10} \mathrm{~K}\right)$, models $\mathrm{D}, \mathrm{T}$, and $\mathrm{S}$ have a different local luminosity (see Eq. (9)). Note that in the Newtonian models the energy emission rate from the neutrinosphere and the energy deposition rates do not depend on the observer, because there is no gravitational redshift effect.

In the local frame GR effects increase the energy deposition rate $\dot{E}_{\gamma \bar{v}}$ of models D, T, and S with respect to their Newtonian counterparts (see Table 2). The increase is smaller when restricting the comparison to the "up" region (where the radial component of the momentum deposition vector is positive, $Q^{r}>0$ ). But even in the "worst" case (toroidal model T) GR effects enhance $\dot{E}_{v \bar{v}}^{\text {up }}$ by about $40 \%$. The optimal geometry to release energy in the surroundings of the $\mathrm{BH}$ is that of a thin disk, where the GR enhancement of the energy deposition rate in the "up" region is $x_{v \bar{v}}^{\text {up }}=2.3$ for a local observer and where the energy deposition rate in the "up" volume is larger than for toroidal and spherical geometries. These results also hold for a distant observer (Table 2). The energy deposition by $v \bar{v}$-annihilation is therefore enhanced in GR models compared to the Newtonian treatment, despite the fact that the luminosity $L_{v}^{\infty}$ for an observer at infinity is smaller (Table 1). This in the first moment counterintuitive behaviour can be understood by the relativistic effects, which account for a more intense neutrino radiation field close to the emitting surface, i.e. the local luminosity, which is not
Table 2. Comparison of idealized models: for each pair of models $x_{v \bar{v}} \equiv \dot{E}_{\bar{y}}(\mathrm{M} 1) / \dot{E}_{\bar{v}}(\mathrm{M} 2)$ denotes the ratio of the total annihilation rates in models M1 and M2. The superscript "tot" indicates that this ratio is obtained by integrating the local energy deposition rates over the whole grid, while for the superscript "up" the integration is restricted to the "up" region, where the energy released by $v \bar{v}$-annihilation is not trapped by the $\mathrm{BH}$ and may eventually reach a distant observer. The ratio is computed in the local frame or in the frame of an infinitely distant observer. The latter case is indicated by the additional superscript " $\infty$ ".

\begin{tabular}{cc|cccc}
\hline \hline M1 & M2 & $x_{v \bar{\nu}}^{\text {tot }}$ & $x_{v \bar{\nu}}^{\text {up }}$ & $x_{v \bar{v}}^{\text {tot, } \infty}$ & $x_{v \bar{v}}^{\text {up, },}$ \\
\hline D & DN & 3.6 & 2.3 & 2.4 & 1.9 \\
T & TN & 4.1 & 1.4 & 2.8 & 1.2 \\
S & SN & 1.8 & 1.8 & 1.3 & 1.3 \\
T & D & 1.2 & 0.48 & 1.2 & 0.48 \\
S & D & 0.22 & 0.44 & 0.23 & 0.36 \\
TN & DN & 1.1 & 0.79 & 1.1 & 0.79 \\
SN & DN & 0.44 & 0.55 & 0.44 & 0.55 \\
DA1 & D & 1.0 & 1.0 & 1.1 & 1.0 \\
DL6.5 & D & 0.56 & 1.0 & 0.69 & 1.0 \\
\hline A2B & REF & 0.92 & 0.61 & 0.81 & 0.61 \\
AB & REF & 1.1 & 0.45 & 0.92 & 0.45 \\
A.5B & REF & 1.7 & 0.39 & 1.2 & 0.39 \\
RI4.5 & REF & 1.5 & 1.3 & 1.3 & 1.2 \\
RI3 & REF & 2.3 & 1.3 & 1.8 & 1.3 \\
TEMP & AB & 5.3 & 3.0 & 4.7 & 2.9 \\
\hline
\end{tabular}

reduced by gravitational redshift, is always higher or at least equal to the Newtonian counterpart of a GR model.

The enhancement of the energy deposition rate due to GR effects depends strongly on the position, and becomes negligible at sufficiently large distances from the BH (Fig. 4, left panel), where relativistic effects are unimportant. In case of the disk models (D and DN), Fig. 2 (upper panels) and Fig. 4 (left panel) show that GR effects enhance the energy deposition rate near the symmetry axis $\left(\theta \lesssim 30^{\circ}\right)$ by a factor of $\gtrsim 10$ up to $r \sim 10 \mathrm{~km}$, and they still increase the energy release by a factor of $\sim 2$ at $r \sim 30 \mathrm{~km}$.

\subsubsection{Influence of the neutrinosphere geometry}

According to Table 2, the energy deposition rate by $v \bar{v}$-annihilation in the "up" region measured by an observer at infinity is largest for a neutrinosphere having the geometry of a thin disk (model D; $\dot{E}_{v \bar{v}}^{\text {up, }}=2.3 \times 10^{48} \mathrm{erg} \mathrm{s}^{-1}$; Table 1), while it is smallest for a neutrinosphere of spherical shape (model S; $\dot{E}_{\gamma \bar{\gamma}}^{\text {up } \infty}=0.83 \times 10^{48} \mathrm{erg} \mathrm{s}^{-1}$; Table 1). This finding holds for the corresponding Newtonian models (DN and SN), too. This is a remarkable result, as in the spherical models $Q^{r}>0$ everywhere on the computational grid (for models $\mathrm{S}$ and $\mathrm{SN}$ the $v \bar{v}$-annihilation integrals in the "up" regions are equal to the corresponding integrals in the "tot" region, respectively; see Table 1).

The influence of the neutrinosphere geometry on the energy deposition rate can also be studied by considering toroidal models with different meridional cross sections. All our toroidal models have an elliptical meridional cross section characterized by its eccentricity (Table 1). Model REF (an infinitely thin disk model with an eccentricity $e=1$ ) serves as the reference model, which is compared to models $\mathrm{A} 2 \mathrm{~B}$ ( $e=\sqrt{3} / 2$, oblate), AB ( $e=0$, circular) and A.5B ( $e=\sqrt{3} / 2$, prolate), respectively. These four models have the same Newtonian luminosity $L_{v}^{\mathrm{N}}$, and the center of their meridional cross sections or the mid-points of their disks are all located at $r_{\mathrm{c}} \approx 18 \mathrm{~km}$ in the equatorial plane. 

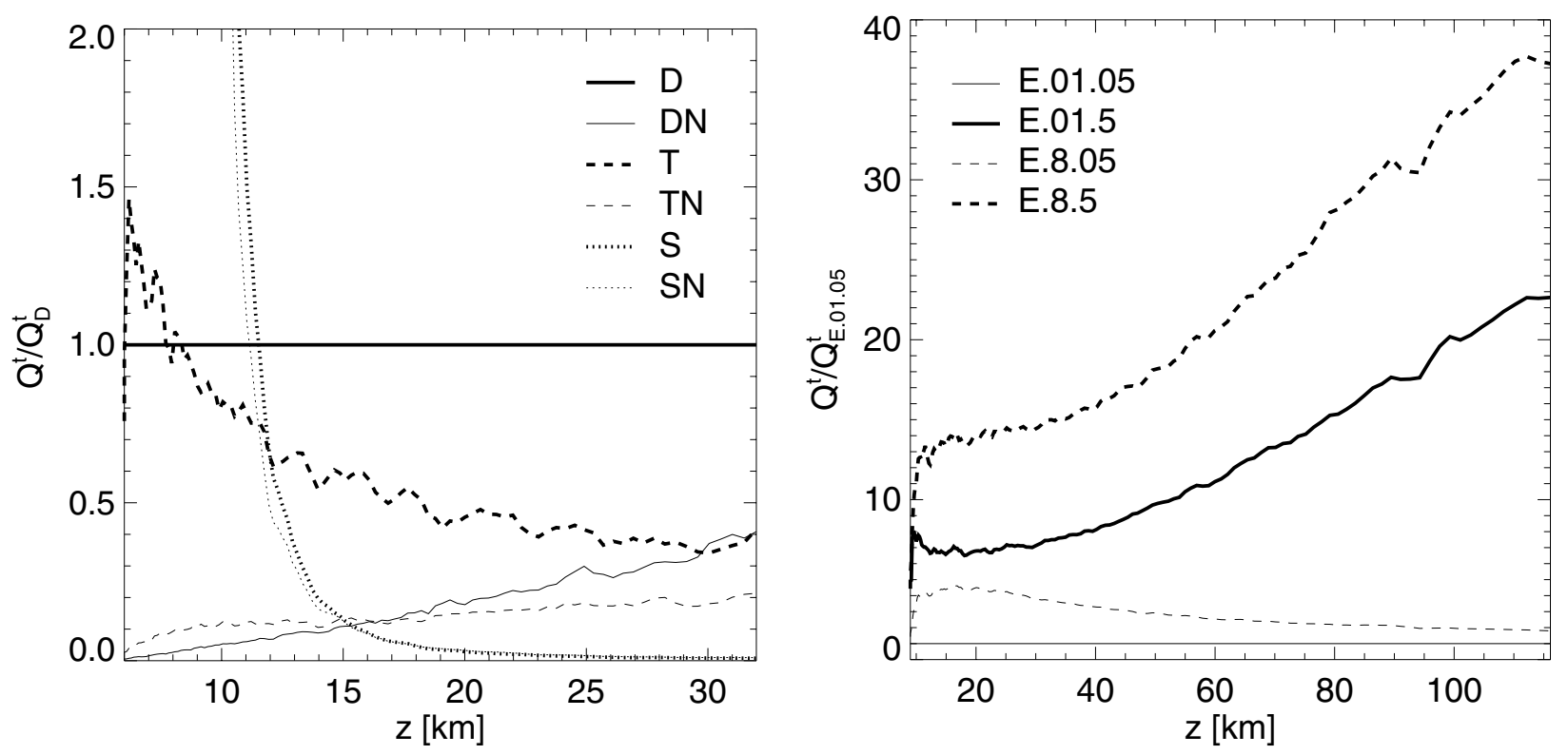

Fig. 4. Energy component of the annihilation rate 4-vector along the $z$-axis for models with idealized neutrinosphere geometries (left panel, see Table 1), and for models where the neutrinospheres are calculated from equilibrium torus models (right panel, see Table 3). The values are normalized to the energy component of the annihilation rate 4-vector along the $z$-axis of models D and E.01.05, respectively.

Compared to the thin disk model REF, the other torus models are all less efficient in depositing energy in the "up" region (Table 2). By inspection of Table 1 one recognizes that the energy deposition rate in the "up" region measured locally and by a distant observer $\dot{E}_{v \bar{v}}^{\text {up }, \infty}$ decreases when the meridional cross section becomes more prolate. It is smallest for model A.5B, which is the most prolate model. At the same time this model is the most efficient one in the total energy release $\left(x_{v \bar{v}, \mathrm{~A} .5 \mathrm{~B}}^{\text {tot } \infty}=1.2>1\right)$. This at first glance surprising behavior can be explained by the fact that a thin disk emits a large fraction of the neutrinos in directions parallel to the symmetry axis, and hence just above and below the thin disk. Consequently, $v \bar{v}$-encounters are less frequent close to the $\mathrm{BH}$ than in a source of toroidal shape. Although infinitely thin disks are a mathematical idealization more than a natural case, our results nevertheless imply that a distant observer receives more energy from systems which have a more oblate structure of a toroidal accretion disk.

Another geometrical aspect of relevance is the size of the innermost radius of the disk $\left(r_{\mathrm{in}}\right)$. To study the dependence of the energy deposition rate on this parameter, we consider the thin disk models RI4.5 $\left(r_{\text {in }}=4.5 M\right)$ and RI3 $\left(r_{\text {in }}=3 M\right)$, which both have an innermost radius smaller than that of model REF ( $r_{\text {in }}=$ $6 M$ ), as can be seen from Table 1, but have the same Newtonian luminosity $L_{v}^{\mathrm{N}}$ and Newtonian surface area. Independent of the region of integration ("up" or "total") and of the observer (local or distant), we find that the smaller is $r_{\text {in }}$, the larger is the energy released by $v \bar{v}$-annihilation (Table 2 ).

Finally, we consider model TEMP (Table 1) to further investigate the influence of the neutrinosphere geometry on the energy deposition rate. This model is identical to model AB, except that in model TEMP only the inner half of the torus (up to a distance of $8 M$ from the symmetry axis) is assumed to emit neutrinos. Thus, in order to have the same Newtonian luminosity $L_{v}^{\mathrm{N}}$ in both models, the neutrinosphere temperature has to be increased from $T_{\mathrm{C}}=5 \times 10^{10} \mathrm{~K}$ to $T_{\mathrm{C}}=6.1 \times 10^{10} \mathrm{~K}$, because the emitting surface is smaller. The more concentrated neutrino radiation field therefore leads to a strong overall increase of the energy deposition rate $\left(x_{\bar{v}} \gtrsim 3\right.$; Table 2$)$, in analogy to the models of the previous paragraph, where the innermost disk radius was reduced.

\subsubsection{Influence of the angular momentum of the $\mathrm{BH}$}

The dependence of the energy deposition rate on the angular momentum of the central BH is studied using model DA1, which consists of a disk surrounding a maximally rotating Kerr BH ( $a=1$; Table 1; Fig. 3). Compared to the corresponding nonrotating model D $(a=0)$, we do not find an enhancement of the energy deposition rate, independent of the region of integration and of the location of the observer (Table 2). This result differs from that of Miller et al. (2003), who obtained a factor of $\sim 2$ larger amount of deposited energy when comparing thin accretion disks orbiting a maximally rotating and a non-rotating $\mathrm{BH}$, respectively. This discrepancy arises because we set $r_{\text {in }}=6 \mathrm{M}$ both in model D and DA1, in order to just test the influence of $\mathrm{BH}$ rotation. In contrast, the disks considered by Miller et al. extended from a fixed outer radius $r_{\text {out }}=10 \mathrm{M}$ in towards the $\mathrm{BH}$, i.e. to the innermost stable circular orbit. This results in disks with smaller values of $r_{\text {in }}$ as $a \rightarrow 1$ than in our study, and hence, as we have shown in the last section, an increased energy deposition rate is expected. Table 1 also contains the two models A.5 and $\mathrm{A} 1$, which are equal to our second reference model REF ( $a=0$, and $M_{\mathrm{BH}}=3 M_{\odot}$, in contrast to $M_{\mathrm{BH}}=2 M_{\odot}$ for model D), except that the black hole rotates with $a=0.5$ and $a=1$, respectively. We find that for every considered $\mathrm{BH}$ mass the value of $\dot{E}_{v \bar{v}}^{\text {up }, \infty}$ depends only weakly on the value of $a$.

\subsubsection{Influence of the accretion disk rotation}

In all idealized models discussed up to now with the shape of an infinitely thin disk, a torus, or a sphere, respectively (Table 1), the rotation of the neutrinosphere was neglected in calculating the $v \bar{v}$-annihilation. We now address the consequences of relativistic aberration connected with such rotation on the energy deposition rate. Our idealized rotating disks are assumed to have a uniform Lagrangian angular momentum $l$. In Model DL6.5 the 

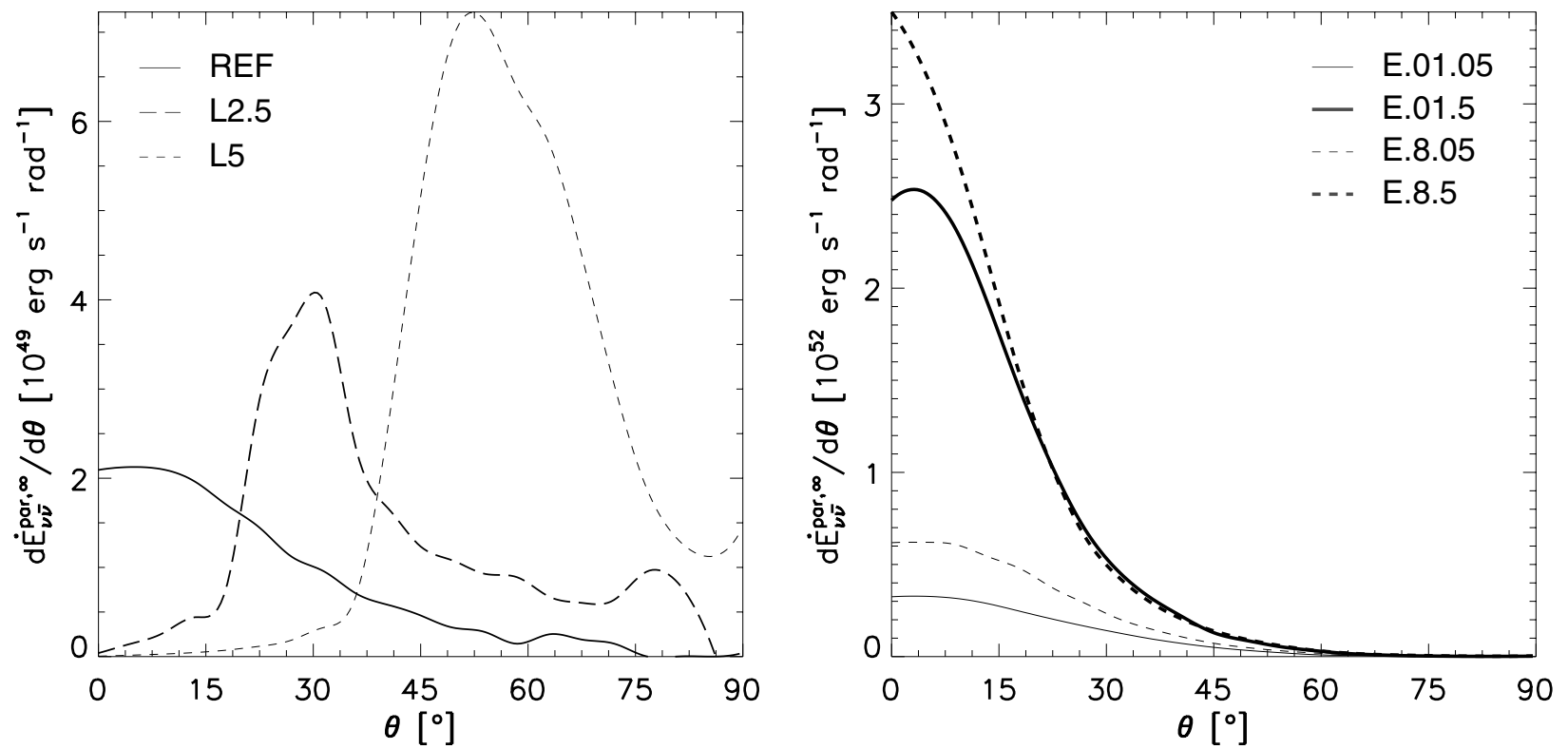

Fig. 5. Energy deposition rate $\dot{E}_{\gamma \bar{\gamma}}^{\mathrm{par}, \infty}$ on a sphere of radius $200 \mathrm{M}$ per unit of Boyer-Lindquist polar angle $\theta$, obtained by taking into account the energy transport to this radius as described by the annihilation rate 4-vector field $Q^{\alpha}$. The left panel shows the results for idealized disk models with different values of the Lagrangian angular momentum $l$, and the right panel the results for the first four equilibrium torus models of Table 3 .

disk is rapidly rotating with the super-Keplerian value $l=6.5 \mathrm{M}$ (corresponding to a cgs value of the specific angular momentum of $l=5.7 \times 10^{16} \mathrm{~cm}^{2} \mathrm{~s}^{-1}$ for the $\left.2 M_{\odot} \mathrm{BH}\right)$, but otherwise the same parameters as in the non-rotating model $\mathrm{D}(l=0)$ are used. For an observer at rest, the (anti)neutrinos are preferentially emitted tangentially to the disk in the direction of rotation. This behavior results in the existence of a non-vanishing $Q^{\phi}$ component and is reflected by the increased size of the filled circles in the vicinity of the disk in Fig. 3. Consequently, neutrinos and antineutrinos collide less frequently with large relative angles, and in the "tot" region of model DL6.5 less energy is deposited than in the non-rotating disk model $\mathrm{D}\left(x_{\bar{y}}^{\text {tot }}<1\right.$; Table 2). However, if we consider the "up" region, this effect is compensated by the slightly increased size of the "up" region in model DL6.5 ( $\left.x_{v \bar{v}}^{\text {up }} \approx 1\right)$, especially in the vicinity of the accretion disk (in model DL6.5 the white line crosses the equatorial plane at a smaller radius than in model D, see, Figs. 2 and 3). The results also hold for an observer at infinity. Hence, independent of the choice of the observer, the influence of the disk rotation on the integrated energy deposition rate in the "up" region is small, which justifies the remarks of Sect. 4.1 regarding the choice of non-rotating reference models. This is also true for intermediate values of $l$ (see Table 1), i.e. in case of the subKeplerian rotation of model L2.5, models DL4 and L4, whose accretion disk approximately rotates at Keplerian speed, and the super-Keplerian model L5 (the three models L2.4, L4, and L5 are not based on model D, but on model REF). Also in case of a toroidal neutrinosphere instead of a disk, the effect of the neutrinosphere rotation on the total annihilation rate measured by an observer at infinity is only small (see the values of $\dot{E}_{\nu \bar{v}}^{\text {up, }}$ for models T and TL4 in Table 1).

We have also studied the influence of the neutrinosphere rotation in case of Newtonian models. For this purpose, we have calculated models DL4N and TL4N (Table 1). When GR effects are ignored we again find that the neutrinosphere rotation does not have an important impact on the total annihilation rate measured at infinity (compare $\dot{E}_{\nu \bar{v}}^{\text {up }, \infty}$ for models DL4N and DN, and TL4N and TN in Table 1).
Despite the weak dependence of the integrated values of the energy deposition rate in the "up" region on the specific angular momentum of the disk, the spatial distribution of the energy deposition rate changes significantly (compare the plots of models D, DL4, and DL6.5 in Figs. 2 and 3). The faster the disk rotation is, the larger are the spatial components of annihilation rate 4-vector tangential to the disk. In addition to that, the energy deposited in the vicinity of the symmetry axis decreases, which is especially visible in model DL6.5.

For the non-rotating model REF and the two rotating models L2.5 and L5 the left panel of Fig. 5 shows the energy $\mathrm{d} \dot{E}_{v \bar{v}}^{\mathrm{par}, \infty} / \mathrm{d} \theta$ arriving at a sphere of radius $200 M$ per unit of time and per unit of Boyer-Lindquist polar angle $\theta$. Numerically, these results are obtained by closely following the approach of Miller et al. (2003). We consider all points of our numerical grid and for each grid point we transport the calculated annihilation rate 4-vector $Q^{\alpha}$ parallel to itself, i.e. we follow geodesics in the direction of the 4-vector, starting at the annihilation point. Excluding the fraction of parallelly transported 4-vectors that eventually hit the black hole or the neutrinosphere, the remaining 4-vectors $Q^{\alpha}$ are transported until they hit the sphere of radius $200 M$ (centered in the system origin). For each such 4 -vector, we take the initial energy component $Q^{t}$, i.e. before the parallel transport, and multiply it by the factor $2 \pi \Delta r \Delta \theta \sqrt{-\operatorname{det}\left(g_{\alpha \beta}\right)}$, in analogy to Eqs. (7) and (8). This way we take into account the 3 -volume and gravitational redshift. Finally, depending on the $\theta$-angle of the 4-vector $Q^{\alpha}$ arriving at the sphere, we create the plots of Fig. 5 by using 20 equally spaced $\theta$-bins.

In case of the rotating thin disk models L2.5 and L5 (see Fig. 5) we obtain results very similar to those of Miller et al. (2003), namely the dominant contribution to $\mathrm{d} \dot{E}_{\gamma \bar{v}}^{\text {par,o }} / \mathrm{d} \theta$ is found to be off-axis and scaling their results to cgs units they quantitatively agree with ours (compare Fig. 8 in their work with the left panel of our Fig. 5). However, the position of the peak strongly depends on the disk rotation, and for non-rotating models the bulk of the energy is deposited in the vicinity of the system axis. In addition to the disk models REF, L2.5, and L5, we have also considered the non-rotating torus model $\mathrm{AB}$ and rotating 
idealized torus models (not shown in this work), and we find the same general behaviour as in case of the disk models.

\subsubsection{Efficiency}

The efficiency of converting radiated neutrino energy into $\mathrm{e}^{+} \mathrm{e}^{-}$pairs by $v \bar{v}$-annihilation is given by the quantity $q_{\nu \bar{v}}^{\text {up }, \infty} \equiv$ $\dot{E}_{v \bar{v}}^{\text {up }, \infty} / L_{v}^{\infty}$, where $L_{v}^{\infty}$ is the sum of the neutrino and antineutrino luminosities at infinity (see Table 1). Values as large as a few tenths of a percent can be reached for $q_{\nu \bar{v}}^{\text {up } \infty}$. Neutrinospheres having the geometry of a thin disk are the most efficient ones in converting neutrino energy into $\mathrm{e}^{+} \mathrm{e}^{-}$-pairs, while neutrinospheres of toroidal shape have the smallest efficiency (see the models $\mathrm{D}, \mathrm{T}$, and $\mathrm{S}$ in Table 1). If GR effects are not taken into account in calculating the energy deposition rate by $v \bar{v}$-annihilation (see models $\mathrm{DN}, \mathrm{TN}$, and $\mathrm{SN}$ ), thin disks are still most efficient, however, now the spherically shaped neutrinosphere is least efficient. Higher disk luminosities increase the deposition efficiency (see the model REF and all models below in Table 1). The efficiency monotonically depends on the inner radius $r_{\text {in }}$ of accretion disks (see models REF, RI4.5, and RI3 in Table 1). When $r_{\text {in }}$ is reduced, the neutrinosphere moves closer to the event horizon of the BH. Although this way the neutrino luminosity measured by an observer at the locations of $v \bar{v}$ annihilation rises (not shown in Table 1), the neutrino luminosity at infinity $L_{v}^{\infty}$ nearly remains the same due to the increased gravitational redshift. Since the energy deposited at infinity $\dot{E}_{v \bar{v}}^{\text {up }, \infty}$ increases, the efficiency becomes larger for smaller values of the inner disk radius $r_{\text {in }}$. An analogous effect can also be seen in the torus model TEMP (see Table 1), where compared to the torus model $\mathrm{AB}$ the accretion torus and Newtonian luminosity $L_{v}^{\mathrm{N}}$ are the same, but the radiating region is closer to the $\mathrm{BH}$. In contrast, up to two significant figures the efficiency exhibits no dependence on the angular momentum of the black hole (see, e.g., model A.5 compared to model REF) and of the disk (see models L2.5, L4, and L5 in Table 1).

\subsection{Equilibrium models}

Next we discuss the $v \bar{v}$-annihilation rate in the vicinity of the neutrinospheres of equilibrium accretion tori computed as described in Sect. 2.2. In all of our equilibrium torus models we keep $s_{\gamma}, r_{\text {in }}$, and $Y_{\mathrm{e}}$ fixed, and therefore we will consider only the influence of the three parameters $\left(M, a, m_{\mathrm{tor}}\right)$ in the following paragraphs.

In contrast to the idealized models, the neutrinosphere and the antineutrinosphere do not coincide in the equilibrium models (Fig. 6), because there is a larger number density of free neutrons than free protons in the equilibrium tori. Hence, the opacity for neutrinos and antineutrinos differs, and consequently the locations of the neutrinosphere and antineutrinosphere, too. Since the matter in the equilibrium tori is neutron rich, the antineutrinosphere is always completely interior to the neutrinosphere. On average it also has a higher temperature (Figs. 6 and 7), i.e. $L_{\bar{v}} \geq L_{v}$. Neither the neutrinosphere nor the antineutrinosphere are isothermal as in case of the idealized models (Fig. 7). Moreover, the torus temperature now depends on the $\mathrm{BH}$ spin and increases, the closer the torus is located around the BH. This effect is ignored in the idealized models.

The energy deposited in the region between the neutrinosphere and the antineutrinosphere cannot be computed by our approach (hence the corresponding region is white in Fig. 6), because in that region transport physics matters, i.e. neutrinos do not propagate freely.

In order to analyze the influence of the torus mass $m_{\text {tor }}$, let us consider the equilibrium models (Table 3) E.01.05, E.01.25 and E.01.5, all of which orbit a slowly rotating BH with $a=$ 0.01, and models E.8.05 and E.8.5, which gird a rapidly rotating BH with $a=0.8$. From Table 4 we infer (i) that a larger torus mass strongly increases the $v \bar{v}$-energy deposition rate, because of a higher neutrino luminosity, and (ii) that this effect is stronger for lower values of $a$. The increase of the energy deposition rate is almost linear with $m_{\text {tor }}$ for high values of $a$, but grows like $m_{\text {tor }}^{0.6}$ for $a=0.01$. Thus, for a distant observer the energy released in the "up" region can be roughly fitted by $\dot{E}_{v \bar{v}}^{\text {up } \infty} \propto \dot{E}_{v \bar{v}}^{\text {up }, \infty}(\mathrm{E} .01 .05)$. $\left(m_{\mathrm{tor}} / M_{\odot}\right)^{0.4 a+0.6}$.

On the other hand, comparing models with the same torus mass but with BHs that have different $a$, e.g. models E.01.05 and E.8.05, shows that larger values of $a$ lead to a larger energy deposition $\left(x_{v \bar{v}}^{\text {up }, \infty}=2.6\right.$; Table 4). The effect of $a$ on the deposition rate is about a factor of 2 smaller for higher torus masses (compare models E.8.5 and E.01.5 in Table 4).

What causes the increased energy deposition rate in case of faster rotating BHs? There are two possibilities: the rotation rate parameter $a$ influences (i) the geometry of the accretion torus and hence the neutrinosphere, and (ii) it affects the (anti)neutrino trajectories through frame dragging. In order to determine the importance of both possibilities, we consider artificial test models where we construct the torus model for a rotating $\mathrm{BH}$ but then disregard the $\mathrm{BH}$ rotation for computing the neutrino geodesics, or vice versa. This means effectively that we consider two different rotation parameters for the same model. The first one, $a_{\mathrm{tor}}$, is used to calculate the equilibrium tori, and hence the neutrinospheres. The second one, $a$, is used to calculate the GR effects on the propagation and annihilation of neutrinos and antineutrinos. Note that the smaller of both values is used to set the inner edge of the computational grid, which is thus located slightly outside the larger of both horizons. Such models are actually inconsistent, because a change in the $\mathrm{BH}$ spin automatically translates into a structural change of the accretion torus. We nevertheless consider them in order to be able to discriminate the influence of the $\mathrm{BH}$ rotation on the $v \bar{v}$-annihilation through its effects on the neutrino trajectories from those effects that result from changes of the torus properties around rotating or nonrotating BHs.

For each of the two parameters, $a$ and $a_{\text {tor }}$, we consider two extreme values, which leads to the four models E.01.1, E.01.1d, E1.1d, and E1.1, respectively (Table 3). As in case of the idealized models (Sect. 4.1), increasing the BH's rotation rate from $a=0.01$ to $a=1$ does not yield any appreciable increase of the energy deposition rate $\left(x_{v \bar{v}}^{\text {up }, \infty} \approx 1\right)$ for both values of $a_{\text {tor }}$ (Table 4$)$. However, a substantial increase $(\sim 60 \%)$ of the energy released in the "up" region (as seen by a distant observer) is found when increasing $a_{\text {tor }}$ from 0.01 to 1 . Thus, we can unambiguously conclude that the increase of the dimensionless angular momentum of the $\mathrm{BH}$ yields a larger energy deposition by $v \bar{v}$-annihilation essentially exclusively because it allows for a smaller innermost radius of the equilibrium accretion torus.

Concerning the influence of the GR effects, a comparison of model E.01.1 with its Newtonian counterpart E.01.1N (Table 3) shows that GR effects on the neutrino propagation increase $\dot{E}_{v \bar{v}}^{\text {up }}$ in case of the equilibrium models only by $\sim 20 \%$. This enhancement of the energy deposition rate is similar to that obtained for the idealized torus models (see Sect. 4.1).

In analogy to the idealized models, we also computed the energy deposition rate distribution on a sphere of radius $200 M$ per 

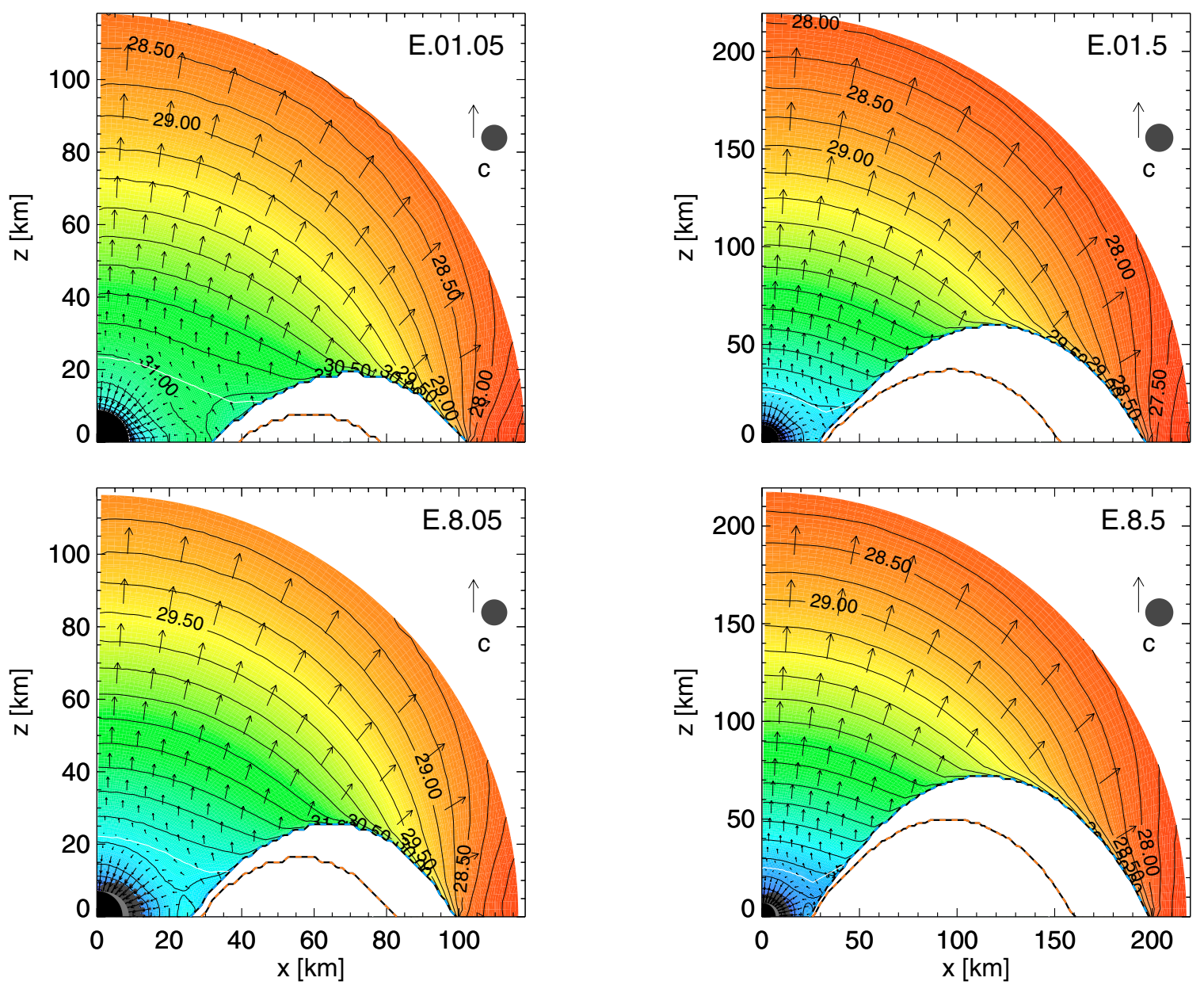

$\lg Q^{t}\left[\operatorname{erg~s}^{-1} \mathrm{~cm}^{-3}\right]$

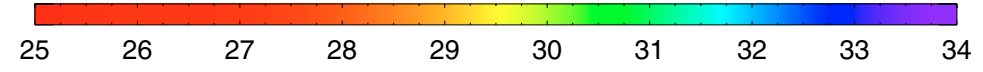

Fig. 6. Local annihilation rates for neutrinosphere geometries calculated from four equilibrium torus models (see Table 3). The visualization method is the same as in Figs. 2 and 3, but here the neutrinospheres for $v_{\mathrm{e}}$ (blue, long dashes) and $\bar{v}_{\mathrm{e}}$ (red, short dashes) do not coincide. Note that the accretion tori of the four models shown here rotate, however the spatial components of the annihilation rate 4-vector perpendicular to the displayed $x-z$-plane are very small and visible only in the close vicinity of the black hole. See the electronic edition for a color version of the figure.

Table 3. Properties of equilibrium torus models, where the location of the $v_{\mathrm{e}}$-sphere differs from that of the $\bar{v}_{\mathrm{e}}$-sphere. Besides the quantities already defined in Table 1, the models here are characterized by the mass $m_{\mathrm{tor}}$ of the accretion torus, and the mass $M_{\mathrm{tor}}$ and angular momentum $a_{\mathrm{tor}}$ of the $\mathrm{BH}$ used to calculate the structure of the accretion torus. In most models the values of the last two quantities are equal to the values of the mass $M$ and angular momentum $a$ of the BH that we use to determine the neutrino geodesics. However, in the two models E.01.1d and E1.1d we have chosen $a_{\text {tor }} \neq a$, in order to discriminate between the influence of the black hole rotation on the accretion torus and on the neutrino trajectories (see Sect. 4.2). For the Newtonian model E.01.1N the table shows $M \neq M_{\text {tor }}$ (and also $a \neq a_{\text {tor }}$ ), because the neutrino trajectories are evaluated without GR effects. All models in this table have the same photon entropy $s_{\gamma}=1$, the same inner equatorial torus radius $r_{\text {in }}=4.1 M$, and the same electron fraction $Y_{\mathrm{e}}=0.1$ (see Sect. 2.2). Moreover, the effects of the torus angular momentum $l$ are included in the evaluation of the $v \bar{v}$-annihilation. Note that $l$ is derived from the set of six quantities $\left(M, a, m_{\mathrm{tor}}, s_{\gamma}, r_{\mathrm{in}}, Y_{\mathrm{e}}\right)$ that fully specify a torus in rotational equilibrium.

\begin{tabular}{|c|c|c|c|c|c|c|c|c|c|c|c|c|c|}
\hline $\begin{array}{c}\text { Model } \\
\text { name }\end{array}$ & $\begin{array}{l}M \\
M_{\odot} \\
\end{array}$ & $a$ & $\begin{array}{c}M_{\text {tor }} \\
M_{\odot} \\
\end{array}$ & $\begin{array}{c}a_{\mathrm{tor}} \\
M \\
\end{array}$ & $\begin{array}{c}m_{\text {tor }} \\
M_{\odot}\end{array}$ & $\begin{array}{c}l \\
M_{\mathrm{GR}} \\
\end{array}$ & $\begin{array}{c}L_{v}^{\infty} \\
10^{54} \mathrm{erg} \mathrm{s}^{-1} \\
\end{array}$ & $\begin{array}{c}\dot{E}_{\nu \bar{v}}^{\text {tot }} \\
10^{52} \mathrm{erg} \mathrm{s}^{-1} \\
\end{array}$ & $\begin{array}{c}\dot{E}_{v \bar{v}}^{\mathrm{up}} \\
10^{52} \mathrm{erg} \mathrm{s}^{-1} \\
\end{array}$ & $\begin{array}{c}\dot{E}_{v \bar{v}}^{\mathrm{tot}, \infty} \\
10^{52} \mathrm{erg} \mathrm{s}^{-1} \\
\end{array}$ & $\begin{array}{c}\dot{E}_{v \bar{v}}^{\text {up, }} \\
10^{52} \mathrm{erg} \mathrm{s}^{-1} \\
\end{array}$ & $\begin{array}{l}q_{\nu \bar{v}, \infty}^{\text {tot }} \\
10^{-2} \\
\end{array}$ & $\begin{array}{l}q_{\nu \bar{v}}^{\text {up } \infty} \\
10^{-2}\end{array}$ \\
\hline E.01.05 & 3 & 0.01 & 3 & 0.01 & 0.05 & 3.95 & 0.32 & 0.83 & 0.40 & 0.59 & 0.36 & 1.9 & 1.1 \\
\hline E.01.5 & 3 & 0.01 & 3 & 0.01 & 0.5 & 3.98 & 0.99 & 6.4 & 3.0 & 4.6 & 2.7 & 4.7 & 2.7 \\
\hline E.8.05 & 3 & 0.8 & 3 & 0.8 & 0.05 & 3.48 & 0.44 & 2.9 & 1.0 & 1.9 & 0.93 & 4.3 & 2.1 \\
\hline E.8.5 & 3 & 0.8 & 3 & 0.8 & 0.5 & 3.53 & 1.1 & 10 & 4.0 & 7.4 & 3.7 & 6.6 & 3.3 \\
\hline E.01.1 & 3 & 0.01 & 3 & 0.01 & 0.1 & 3.96 & 0.51 & 2.1 & 1.0 & 1.5 & 0.92 & 2.9 & 1.8 \\
\hline E.01.1d & 3 & 0.01 & 3 & 1 & 0.1 & 3.96 & 0.62 & 4.5 & 1.7 & 2.9 & 1.5 & 4.6 & 2.4 \\
\hline E1.1d & 3 & 1 & 3 & 0.01 & 0.1 & 3.96 & 0.51 & 2.0 & 1.0 & 1.6 & 0.94 & 3.1 & 1.8 \\
\hline E1.1 & 3 & 1 & 3 & 1 & 0.1 & 3.48 & 0.62 & 4.9 & 1.7 & 3.3 & 1.5 & 5.3 & 2.4 \\
\hline E.01.25 & 3 & 0.01 & 3 & 0.01 & 0.25 & 3.98 & 0.79 & 4.6 & 2.1 & 3.2 & 1.9 & 4.1 & 2.4 \\
\hline E. $01.1 \mathrm{~N}$ & 0 & 0 & 3 & 0.01 & 0.1 & 3.96 & 0.57 & 0.90 & 0.76 & 0.90 & 0.76 & 1.6 & 1.3 \\
\hline
\end{tabular}




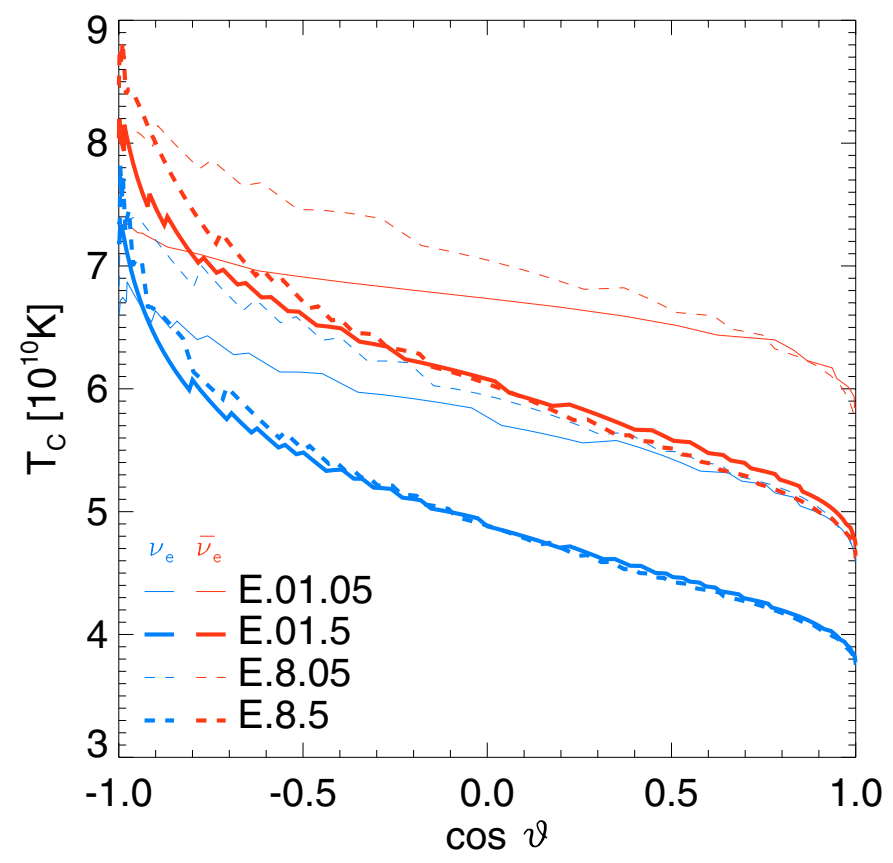

Fig. 7. Temperature profiles of the neutrinospheres of the models in Fig. 6 in the comoving frame versus the angle $\vartheta$, which is defined as follows: if $r_{\mathrm{c}}$ is the arithmetic mean of the innermost $\left(r_{\mathrm{in}}\right)$ and outermost $\left(r_{\text {out }}\right)$ radius where the neutrinosphere intersects the equatorial plane, then given a point $C$ on the neutrinosphere the angle $\vartheta$ is enclosed by the legs $\overline{r_{\mathrm{c}} r_{\mathrm{out}}}$ and $\overline{r_{\mathrm{c}} C}$, respectively. The blue (red) lines denote the temperature of the neutrinosphere (antineutrinosphere). See the electronic edition for a color version of the figure.

polar angle $\theta$ for equilibrium models (see right panel of Fig. 5). However, in contrast to the idealized models, the bulk of the energy is always deposited near the system axis. Hence, the result of Miller et al. (2003), i.e. that the main contribution to the energy deposition rate comes from the off-axis region, is valid only for idealized models (see Sect. 4.1.4), but in equilibrium torus models the energy deposition along the symmetry axis is clearly dominant.

The efficiency $q_{v \bar{v}}^{\mathrm{up}, \infty}$ increases for our equilibrium models both with the torus mass and with $a$, reaching values of 1-3 per cent (Table 3) in agreement with the results of Jaroszynski (1996) for his models with specific entropies $\gtrsim 8$, and with Setiawan et al. (2004) for their models with the largest $\alpha$-viscosity. The increase with $a$ is a structural effect, because for larger values of $a$ the torus is on average closer to the rotation axis and hotter, i.e. the neutrino luminosity is higher. Therefore the neutrino density is higher near the system axis and hence a larger fraction of neutrinos and antineutrinos annihilate with each other. The efficiencies of the equilibrium models are $\sim 10$ times larger than those of the idealized models, because both the temperatures (Fig. 7) and the surface areas of their neutrinospheres are larger.

\section{Conclusions}

The main goal of this paper is to study generic properties of the process of energy deposition by neutrino-antineutrino $(v \bar{v})$ annihilation in the vicinity of systems consisting of a central stellarmass black hole $(\mathrm{BH})$ and an accretion disk or torus surrounding it. Assuming that the thermodynamic conditions in the accretion flow are such that a copious flux of $v \bar{v}$-pairs can be produced, we have performed a systematic parameter study of the influence of
Table 4. Comparison of the equilibrium torus models. The table is structured like Table 2, but the models shown here are models listed in Table 3.

\begin{tabular}{cc|cccc}
\hline \hline M1 & M2 & $x_{v \bar{v}}^{\text {tot }}$ & $x_{v \bar{v}}^{\text {up }}$ & $x_{v \bar{v}}^{\text {tot, }}$ & $x_{v \bar{v}}^{\text {up, }}$ \\
\hline E.01.5 & E.01.05 & 7.7 & 7.5 & 7.8 & 7.5 \\
E.8.5 & E.8.05 & 3.4 & 4.0 & 3.9 & 4.0 \\
E.8.05 & E.01.05 & 3.5 & 2.5 & 3.2 & 2.6 \\
E.8.5 & E.01.5 & 1.6 & 1.3 & 1.6 & 1.4 \\
\hline E1.1d & E.01.1 & 1.0 & 1.0 & 1.1 & 1.0 \\
E1.1 & E.01.1d & 1.1 & 1.0 & 1.1 & 1.0 \\
E.01.1d & E.01.1 & 2.1 & 1.7 & 1.9 & 1.6 \\
E1.1 & E1.1d & 2.5 & 1.7 & 2.1 & 1.6 \\
\hline E.01.25 & E.01.05 & 5.5 & 5.3 & 5.4 & 5.3 \\
E.01.1 & E.01.1N & 2.3 & 1.3 & 1.7 & 1.2 \\
\hline
\end{tabular}

the mass and dimensionless angular momentum parameter of the $\mathrm{BH}$, of the shape and thermal properties of the neutrinosphere, and of the importance of different general relativistic (GR) effects on the amount of energy released by $v \bar{v}$-annihilation.

On the one hand we considered idealized models having an isothermal neutrinosphere of prescribed temperature and geometry, and on the other hand non-selfgravitating, axisymmetric equilibrium tori bound to a central $\mathrm{BH}$ of given properties. In the latter models the neutrinospheres of neutrinos and antineutrinos do not coincide, because they are computed for opacities of $v_{\mathrm{e}}$ and $\bar{v}_{\mathrm{e}}$ that differ due to absorptions on free neutrons or free protons, respectively (similar to the work of Jaroszynski 1993, 1996). Using both sets of models we numerically calculated the annihilation rate energy-momentum 4-vector. For this purpose, we constructed the local neutrino distribution by ray-tracing neutrino trajectories in a Kerr space-time using GR geodesics. Our study is a generalization of the work of Miller et al. (2003) to axisymmetric neutrinospheres of different shapes, which are not necessarily spatially coincident for $v_{\mathrm{e}}$ and $\bar{v}_{\mathrm{e}}$.

We considered three different neutrinosphere geometries in our set of idealized models covering cases that may be encountered in astrophysical systems: infinitely thin disks, tori, and spheres. Infinitely thin disks are mathematical idealizations. However, they are investigated as the limiting case of very oblate tori. For this reason they have been widely used in the literature. By comparing with corresponding Newtonian models, where the influence of the gravitational field on the neutrino propagation is neglected, we have found that for an observer at infinity GR effects enhance the energy deposition rate by a factor of 2 in the non-rotating, thin disk case in agreement with Asano \& Fukuyama (2001). In the other two cases the influence of GR effects enhances the annihilation rate only by $\approx 25 \%$.

Independent of whether GR effects are included, the energy deposition rate that may lead to the acceleration of relativistic outflow is largest for infinitely thin disks. For more prolate tori the energy deposition rate drops in comparison with a disk that has the same neutrino luminosity, because a sizeable fraction of the energy deposited by $v \bar{v}$-annihilation is swallowed by the $\mathrm{BH}$. Spherical neutrinospheres are the least favorable geometry for a large total annihilation rate. Accretion disks encountered in gamma-ray burst (GRB) scenarios are likely to be geometrically thick, in which case the geometry of their neutrinospheres is torus-like. For such a situation neglecting GR effects in the evaluation of $v \bar{v}$-annihilation is a good approximation.

We also analyzed the influence of the dimensionless rotation parameter $a$ of the $\mathrm{BH}$, and we confirm the findings of 
Miller et al. (2003) that modifying $a$ without changing the innermost disk radius (i.e., leaving the thin disk geometry unchanged) has no effect on the amount of released $v \bar{v}$-annihilation energy. The independence of the results of the $\mathrm{BH}$ rotation holds both for idealized models with toroidal neutrinospheres and for equilibrium accretion torus models. However, in a consistent accretion disk model, the innermost radius of the disk (close to the innermost stable circular orbit) will shrink as $a \rightarrow 1$. We have found that a smaller innermost disk radius leads to a substantial increase of the energy deposition rate, even when the local Newtonian (i.e., GR effects disregarded) neutrino luminosity is the same. This can be understood by the higher neutrino density in the vicinity of a more compact disk and was also found by Miller et al. (2003).

Depending on the mass of the accretion torus, models containing a maximally rotating $\mathrm{BH}(a=1)$ can release roughly a factor of 2 more energy (as measured by a distant observer) by $v \bar{v}$-annihilation than models involving non-rotating BHs. For a small accretion disk mass $\left(\sim 0.05 M_{\odot}\right)$ the energy release in case of a rotating $(a=0.8) \mathrm{BH}$ is more than 2.5 times larger than in case of a non-rotating BH. The difference is smaller for larger torus masses. Considering that low-mass accretion tori rotating around a $\mathrm{BH}$ with intermediate values of the dimensionless angular momentum parameter $(a \sim 0.6-0.8)$ may be typical products of mergers of compact objects (e.g., Ruffert et al. 1997; Setiawan et al. 2004), we have demonstrated that all other GR effects are of moderate importance when calculating the amount of energy released in such systems by $v \bar{v}$-annihilation. However, we point out that the spatial distribution of the released energy exhibits very important differences between Newtonian and GR models: close to the rotation axis and close to the bounding of the "up" region, GR effects can enhance the local energy deposition rate by a factor of $\sim 10$. Thus, a relativistic jet driven by the energy release in a region close to the stagnation surface (which will form in the vicinity of the boundary of the "up" region) should receive a much larger energy input due to GR effects. The question whether this difference in the spatial distribution of the energy deposition is more favorable for producing ultrarelativistic jets can not be addressed by the present work, but requires time-dependent hydrodynamic jet simulations including a detailed description of $v \bar{v}$-annihilation (see below).

In our set of idealized models, a thin disk (sphere) is the most (least) favorable geometry to convert neutrino energy into $\mathrm{e}^{+} \mathrm{e}^{-}$-pairs by $v \bar{v}$-annihilation. Our idealized models yield efficiencies $q_{\nu \bar{v}}^{\text {up, }}$ of only a few tenths of a per cent. Larger efficiencies, of the order of several percent, have been obtained for our more realistic equilibrium models. These findings are in the ballpark of the results obtained by Jaroszynski (1996) (for models with specific entropies $\gtrsim 8$ and Setiawan et al. (2004); for models with the largest $\alpha$-viscosity).

Miller et al. (2003) have pointed out that the main contribution to the energy-momentum deposition rate comes from large polar angles (particularly from regions above the neutrinospheres) and not from regions near the symmetry axis. This behavior, however, results from their restriction to infinitely thin disk models, which are mathematically idealized cases. According to our studies, only such idealized models show this strong enhancement of the annihilation rate towards the equatorial plane, while this effect is absent in the more realistic equilibrium torus models. Hence, this finding of Miller et al. (2003) appears to be of little relevance for the amount of energy that is available to produce a GRB. In any realistic situation the accretion disk/torus will be inflated vertically, i.e. the baryon density will be highest in the equatorial plane and decrease in perpendicular direction, the density scale height of the baryon distribution being larger for large disk masses and high disk temperatures.

As demonstrated in Aloy et al. (2005), who considered similar equilibrium tori as initial models for their hydrodynamic simulations of relativistic jets from $\mathrm{BH}$-torus systems, timedependent numerical simulations are needed in order to determine the feedback of $v \bar{v}$-energy deposition on the torus structure, which, in its turn, can yield a number of highly non-linear relativistic hydrodynamic effects on the outflowing jet (Aloy \& Rezzolla 2006). This also decides about which fraction of the energy deposited by $v \bar{v}$-annihilation is finally useful for accelerating an ultrarelativistic fireball powering a GRB event. This fraction can be different from the annihilation efficiency $q_{\nu \bar{v}}^{\text {up }, \infty}$ defined in the present work. Our equilibrium models produce $v \bar{v}$-annihilation rates $\dot{E}_{\nu \bar{v}}^{\mathrm{up}, \infty} \gtrsim 10^{52} \mathrm{erg} \mathrm{s}^{-1}$ for torus masses $m_{\text {tor }} \gtrsim 0.1 M_{\odot}$. These figures agree within a factor of $2-4$ with the results of dynamical simulations (Setiawan et al. 2006) and they are about one order of magnitude higher than the ones quoted in Jaroszynski (1996) for his models with specific entropies per baryon in the range of 8 to 10 . The difference compared to Jaroszynski's estimates is probably due to the differences in the temperature distribution on the neutrinosphere caused by our somewhat different way of constructing the accretion tori. The neutrino luminosity is very sensitive to the neutrinospheric temperature, and a $\sim 30 \%$ larger value of this temperature can account for a factor of more than 10 larger total $v \bar{v}$-annihilation rate. Considering a certain accretion disk mass and its corresponding neutrino luminosity and annihilation efficiency means an upper bound of the physically likely situation, in which the neutrino luminosity decreases with time as the mass of the disk decreases. However, we remark that the time evolution of the $v \bar{v}$-annihilation rate and the annihilation efficiency can be non-monotonic and does not necessarily decrease with time as demonstrated by Setiawan et al. (2006).

When attempting to link our present results to GRB observations, we must therefore be aware of the restrictions stated in the previous paragraph and in particular of the fact that any reliable estimate of the fraction of the $v \bar{v}$-annihilation energy that drives an ultrarelativistic fireball and the determination of the collimation of such outflow requires hydrodynamic simulations (see also Janka et al. 2006). So we conclude with caution that the energy release by $v \bar{v}$-annihilation in some of our models (especially those having the most massive tori or large values of $a$ ) could be sufficient even to fuel the most distant and most powerful short GRB discovered so far (GRB060121, $E_{\gamma} \approx 3 \times$ $10^{51} \mathrm{erg}$; de Ugarte Postigo et al. 2006) without the need of invoking either ultra-intense magnetic fields $\left(B>10^{16} \mathrm{G}\right)$ or a different progenitor class.

Finally, we point out that Aloy et al. employed a simple fit with time-independent geometry to describe the energy deposition by $v \bar{v}$-annihilation above the poles of a stellar-mass $\mathrm{BH}$ in their relativistic hydrodynamic jet simulations. Preferably, future numerical simulations should include a time-dependent treatment of the energy deposition by $v \bar{v}$-annihilation using the refined methods employed in this work.

Acknowledgements. M.A.A. is a Ramón y Cajal Fellow of the Spanish Ministry of Education and Science. He also acknowledges partial support from the Spanish Ministry of Education and Science (AYA2004-08067-C03-C01). This work has been supported in part by the Sonderforschungsbereich-Transregio 7 "Gravitationswellenastronomie" of the German Science Foundation (DFG). 


\section{Appendix A: Some technical details of the calculation of the annihilation rate}

Here we describe in detail the evaluation of Eq. (1), which is a natural generalization of the formula for the energy component $Q_{i}^{t}$ given in Ruffert et al. (1997).

We express the neutrino 3-momentum $\bar{p}$ (analogous expressions hold for the antineutrino quantities which are represented by primed variables) in terms of the energy $E=|\bar{p}|$ and the unit vector in the momentum direction $\bar{n}=\bar{p} / E$. The distribution function is defined as

$f \equiv f(t, \bar{x}, E, \bar{n}):=\frac{h^{3}}{g} \frac{\mathrm{d} N}{\mathrm{~d} V_{x} \mathrm{~d} V_{p}}$,

where $h$ is Planck's constant, and $g$ is the statistical weight ( $g=$ 1 for neutrinos and antineutrinos). Using $\mathrm{d}^{3} p=E^{2} \mathrm{~d} E \mathrm{~d} \Omega$ and defining $\theta$ as the angle between the directions of propagation of the neutrino and antineutrino, Eq. (1) in full detail reads

$$
\begin{aligned}
Q_{i}^{\alpha}= & \frac{1}{4} \frac{\sigma_{0}}{m_{\mathrm{e}}^{2} h^{6}} \\
& \times\left\{\frac{\left(C_{1}+C_{2}\right)_{v_{i} \bar{v}_{i}}}{3} \int_{0}^{\infty} \mathrm{d} E \int_{0}^{\infty} \mathrm{d} E^{\prime}\left(p^{\alpha}+p^{\prime \alpha}\right) E^{3} E^{\prime 3}\right. \\
& \times \oint_{4 \pi} \mathrm{d} \Omega \oint_{4 \pi} \mathrm{d} \Omega^{\prime}(1-\cos \theta)^{2} f_{v_{i}} f_{\bar{v}_{i}} \\
& +C_{3, v_{i} \bar{v}_{i}} m_{\mathrm{e}}^{2} \int_{0}^{\infty} \mathrm{d} E \int_{0}^{\infty} \mathrm{d} E^{\prime}\left(p^{\alpha}+p^{\prime \alpha}\right) E^{2} E^{\prime 2} \\
& \left.\times \oint_{4 \pi} \mathrm{d} \Omega \oint_{4 \pi} \mathrm{d} \Omega^{\prime}(1-\cos \theta) f_{v_{i}} f_{\bar{v}_{i}}\right\}
\end{aligned}
$$

where $p^{\alpha}=(E, \bar{p}), \sigma_{0}=1.76 \times 10^{-44} \mathrm{~cm}^{2}$ is the weak interaction cross section, $m_{\mathrm{e}}$ the electron mass, and finally

$$
\begin{array}{ll}
\left(C_{1}+C_{2}\right)_{v_{\mathrm{e}} \overline{\mathrm{v}}_{\mathrm{e}}} \approx 2.34, \quad & \left(C_{1}+C_{2}\right)_{v_{x} \bar{v}_{x}} \approx 0.50, \\
C_{3, v_{\mathrm{e}} \bar{v}_{\mathrm{e}}} \approx 1.06, & C_{3, v_{x} \bar{v}_{x}} \approx-0.16,
\end{array}
$$

with $x \in\{\mu, \tau\}$.

In order to perform the ray-tracing of the neutrino trajectories a definition of the base vectors spanning the local observer frame is required. Our choice is the same as that of Miller et al. (2003), i.e.

$$
\begin{aligned}
& \left(\boldsymbol{e}_{t}^{\alpha}\right)=\frac{1}{\sqrt{g_{t t}}}(1,0,0,0),\left(\boldsymbol{e}_{r}{ }^{\alpha}\right)=\frac{1}{\sqrt{-g_{r r}}}(0,1,0,0), \\
& \left(\boldsymbol{e}_{\theta}^{\alpha}\right)=\frac{1}{\sqrt{-g_{\theta \theta}}}(0,0,1,0), \\
& \left(\boldsymbol{e}_{\phi}^{\alpha}\right)=\sqrt{\frac{g_{t t}}{g_{t \phi}^{2}-g_{\phi \phi} g_{t t}}}\left(-\frac{g_{t \phi}}{g_{t t}}, 0,0,1\right),
\end{aligned}
$$

where the metric coefficients are given in Eq. (2). Note that Miller et al. used the same signature as Misner et al. (1973) and that the selected base is orthonormal, i.e. $\boldsymbol{e}_{\alpha} \cdot \boldsymbol{e}_{\beta}=\eta_{\alpha \beta}$, where $\eta_{\alpha \beta}$ is the Minkowski metric.

\section{Appendix B: Convergence tests}

We performed a series of convergence tests to check the dependence of the energy component $Q^{t}$ of the annihilation rate 4-vector on the number $N_{\text {rays }}$ of ray-tracing paths for neutrinos and antineutrinos. The results of this test are shown in Fig. B.1. The dots give the values of $Q^{t}$ at selected points for a representative BH-disk configuration. Successively increasing $N_{\text {rays }}$ (gray

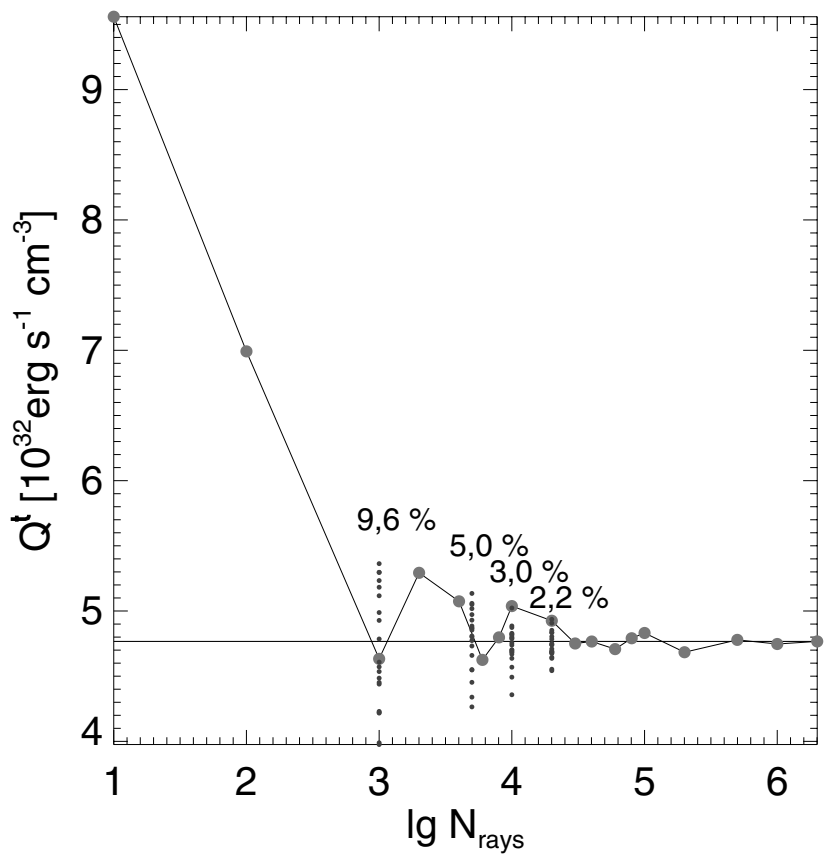

Fig. B.1. Convergence behaviour of the energy component $Q^{t}$ of the annihilation rate 4 -vector for a $M=1 M_{\odot}, a=0$ black hole. The neutrinosphere (for both $v_{\mathrm{e}}$ and $\bar{v}_{\mathrm{e}}$ ) is considered to be a non-rotating isothermal thin disk of temperature $T=10^{11} \mathrm{~K}$ extending from $r_{\text {in }}=$ $3.5 \mathrm{M}$ to $r_{\text {out }}=5.5 \mathrm{M}$. The rate is computed at the point $(r=3.4 \mathrm{M}$, $\theta=\frac{\pi}{4}$ ) using different numbers $N_{\text {rays }}$ of ray-tracing paths for neutrinos and antineutrinos. The horizontal line marks the asymptotic value of $Q^{t}$ as $N_{\text {rays }} \rightarrow \infty$.

filled circles connected by the black, solid line) we found that the value of $Q^{t}$ is converged to an accuracy better than $\sim 3 \%$ for $N_{\text {rays }}>10^{4}$. When calculating $Q^{t}$ repeatedly for four selected values of $N_{\text {rays }}$ the results scatter statistically due to the random procedure used for picking the initial direction of the ray-tracing paths for the (anti)neutrinos. The corresponding relative error is shown in Fig. B.1, too. Since all the simulations presented in this publication have been performed with $N_{\text {rays }}=20000$, the relative error of the calculated annihilation rates $Q^{t}$ is about $2 \%$. This accuracy slightly varies depending on where the annihilation rate is calculated. Analogous tests were also performed for the spatial components of the annihilation rate 4-vector, which show a similar convergence behaviour.

A second series of convergence tests was computed, in order to check the dependence of the total annihilation rate on the grid resolution. In this context model REF, which was simulated on a computational grid of $N_{r}=110$ and $N_{\theta}=100$ points, was recalculated on a grid with twice as many grid points in each coordinate direction (i.e., $N_{r}=220$, and $N_{\theta}=200$ ). Doubling the resolution the value of $\dot{E}_{v \bar{v}}^{\text {tot, } \infty}$ defined in Eq. (7) changes from $6.19 \times 10^{49} \mathrm{erg} \mathrm{s}^{-1}$ (coarse grid) to $6.21 \times 10^{49} \mathrm{erg} \mathrm{s}^{-1}$ (finer grid), which is a small difference of $0.3 \%$.

Finally, we investigated the dependence of the results on the size of the grid, i.e. on the value of the outer grid radius $r_{\mathrm{g}, \text { out }}$. Again using model REF, we increased the outer radius from its standard value $r_{\mathrm{g}, \text { out }}=48.75 \mathrm{~km}$ to $r_{\mathrm{g}, \text { out }}^{\prime}=270 \mathrm{~km}$. The resulting difference in $\dot{E}_{v \bar{v}}^{\mathrm{tot}, \infty}$ is $2.5 \times 10^{48} \mathrm{erg} \mathrm{s}^{-1}$, corresponding to a relative error of $\sim 4 \%$. This error is also representative for the other models (note that there is negligible energy deposition for $r>r_{\mathrm{g}, \text { out }}^{\prime}$ ). 


\section{References}

Abramowicz, M., Jaroszynski, M., \& Sikora, M. 1978, A\&A, 63, 221

Aloy, M. A., \& Rezzolla, L. 2006, ApJ, 640, L115

Aloy, M. A., Müller, E., Ibáñez, J. M., Martí, J. M., \& MacFadyen, A. 2000, ApJ, 531, L119

Aloy, M. A., Janka, H.-T., \& Müller, E. 2005, A\&A, 436, 273

Asano, K., \& Fukuyama, T. 2000, ApJ, 531, 949

Asano, K., \& Fukuyama, T. 2001, ApJ, 546, 1019

Burrows, A., \& Lattimer, J. M. 1986, ApJ, 307, 178

Chen, W.-X., \& Beloborodov, A. M. 2006 [arXiv: astro-ph/0607145]

Cooperstein, J., van den Horn, L. J., \& Baron, E. 1986, ApJ, 309, 653

de Ugarte Postigo, A., Castro-Tirado, A. J., Guziy, S., et al. 2006

[arXiv: astro-ph/0605516]

Di Matteo, T., Perna, R., \& Narayan, R. 2002, ApJ, 579, 706

Eichler, D., Livio, M., Piran, T., \& Schramm, D. N. 1989, Nature, 340, 126

Font, J. A., \& Daigne, F. 2002, MNRAS, 334, 383

Goodman, J., Dar, A., \& Nussinov, S. 1987, ApJ, 314, L7

Janka, H.-T. 2001, A\&A, 368, 527

Janka, H.-T., \& Ruffert, M. 2002, in Stellar Collisions, Mergers and their Consequences, ed. M. M. Shara, ASP Conf. Ser., 263, 333

Janka, H.-T., Eberl, T., Ruffert, M., \& Fryer, C. L. 1999, ApJ, 527, L39

Janka, H.-T., Aloy, M.-A., Mazzali, P. A., \& Pian, E. 2006, ApJ, 645, 1305

Jaroszynski, M. 1993, Acta Astron., 43, 183

Jaroszynski, M. 1996, A\&A, 305, 839

Kohri, K., \& Mineshige, S. 2002, ApJ, 577, 311
Lee, W. H., Ramirez-Ruiz, E., \& Page, D. 2004, ApJ, 608, L5

Lee, W. H., Ramirez-Ruiz, E., \& Granot, J. 2005a, ApJ, 630, L165

Lee, W. H., Ramirez-Ruiz, E., \& Page, D. 2005b, ApJ, 632, 421

MacFadyen, A., \& Woosley, S. E. 1999, ApJ, 524, 262

Miller, W. A., George, N. D., Kheyfets, A., \& McGhee, J. M. 2003, ApJ, 583, 833

Misner, C. W., Thorne, K. S., \& Wheeler, J. A. 1973, Gravitation (San Francisco: W.H. Freeman and Co.)

Mochkovitch, R., Hernanz, M., Isern, J., \& Martin, X. 1993, Nature, 361, 236

Narayan, R., Piran, T., \& Kumar, P. 2001, ApJ, 557, 949

Oechslin, R., \& Janka, H.-T. 2006, MNRAS, 368, 1489

Piran, T. 2005, Rev. Mod. Phys., 76, 1143

Popham, R., Woosley, S. E., \& Fryer, C. 1999, ApJ, 518, 356

Riffert, H., Ruder, H., Nollert, H.-P., \& Hehl, F. W. 1998, Relativistic Astrophysics (Braunschweig: Vieweg)

Rosswog, S., Ramirez-Ruiz, E., \& Davies, M. B. 2003, MNRAS, 345, 1077

Ruffert, M., \& Janka, H.-T. 1999, A\&A, 344, 573

Ruffert, M., Janka, H.-T., Takahashi, K., \& Schaefer, G. 1997, A\&A, 319, 122

Salmonson, J. D., \& Wilson, J. R. 1999, ApJ, 517, 859

Setiawan, S., Ruffert, M., \& Janka, H.-T. 2004, MNRAS, 352, 753

Setiawan, S., Ruffert, M., \& Janka, H.-T. 2006, A\&A, 458, 553

Shibata, M., Taniguchi, K., \& Uryū, K. 2003, Phys. Rev. D, 68, 084020

Shibata, M., Taniguchi, K., \& Uryū, K. 2005, Phys. Rev. D, 71, 084021

Woosley, S. E. 1993, ApJ, 405, 273 